\title{
CB1 agonism prolongs therapeutic window for hormone replacement in ovariectomized mice
}

\author{
Kun Zhang, ${ }^{1,2}$ Qi Yang, ${ }^{1}$ Le Yang, ${ }^{1}$ Yan-jiao Li, ${ }^{2}$ Xin-shang Wang, ${ }^{1,2}$ Yu-jiao Li,,${ }^{1,2}$ Rui-li Dang, ${ }^{2}$ Shao-yu Guan, ${ }^{2}$ Yan-yan Guo, ${ }^{1}$ \\ Ting Sun, ${ }^{1}$ Yu-mei Wu, ${ }^{2}$ An Liu, ${ }^{1}$ Yan Zhang, ${ }^{1}$ Shui-bing Liu, ${ }^{2}$ and Ming-gao Zhao ${ }^{1,2}$ \\ 'Department of Pharmacy, Precision Pharmacy and Drug Development Center, Tangdu Hospital, Fourth Military Medical University, Xi'an, Shaanxi, China. ²Department of Pharmacology, School of Pharmacy, \\ Fourth Military Medical University, Xi'an, Shaanxi, China.
}

\begin{abstract}
Hormone therapy (HT) is reported to be deficient in improving learning and memory in older postmenopausal women according to recent clinical studies; however, the reason for failure is unknown. A "window of opportunity" for estrogen treatment is proposed to explain this deficiency. Here, we found that facilitation of memory extinction and long-term depression by $17 \beta$-estradiol (E2) was normal in mice 1 week after ovariectomy ( $0 \mathrm{VX}_{\mathrm{ST}}$ ), but it was impaired in mice 3 months after ovariectomy $\left(\mathrm{OVX}_{\mathrm{LT}}\right)$. High-throughput sequencing revealed a decrease of miR-221-5p, which promoted cannabinoid receptor 1 (CB1) ubiquitination by upregulation of Neurl1a/b in E2-treated $\mathrm{OVX}_{\mathrm{LT}}$ mice. Blood samples from postmenopausal women aged 56-65 indicated decreases of miR-221-5p and 2-arachidonoylglycerol compared with samples from perimenopausal women aged 46-55. Replenishing of miR-221-5p or treatment with a CB1 agonist rescued the impairment of fear extinction in E2-treated $\mathrm{OVX} \mathrm{LT}_{\mathrm{LT}}$ mice. The present study demonstrates that an $\mathrm{HT}$ time window in mice can be prolonged by cotreatment with a CB1 agonist, implying a potential strategy for HT in long-term menopausal women.
\end{abstract}

\section{Introduction}

Natural menopause is a gradual process that occurs for most women between the ages of 47 and 55 (1). Although hormone therapy (HT) is used as an effective treatment for the impairment of learning and memory in animal models and in menopausal humans (2), clinical research has shown conflicting and controversial results with HT. The Women's Health Initiative Memory Study (WHIMS) indicated that conjugated equine estrogens with or without a progestin (medroxyprogesterone acetate) failed to improve cognitive function when compared with placebo among postmenopausal women aged 65 or older, and an increased risk of cognitive decline even occurred in the conjugated equine estrogens plus progestin group. Thus, HT showed no cognitive advantages and an increased risk for dementia was found in women 65 years of age or older (3). Research indicates that initiation of estrogen treatment at the time of menopause or soon after ovariectomy (OVX) provides a so-called "window of opportunity" for preservation of memory in women, whereas administration of hormone following a considerable delay in time after OVX has little or no beneficial effect on cognition (4). Duration of estrogen deprivation, but not chronologic age, prevents estrogen from enhancing hippocampal synaptic physiology (5). Results from animal and human studies also indicate a window of time (or time window) with respect to preservation of memory in females after OVX or menopause; however, the

Authorship note: KZ, QY, LY, and Yan-JL contributed equally to this work. Conflict of interest: The authors have declared that no conflict of interest exists. Copyright: (5) 2019, American Society for Clinical Investigation.

Submitted: July 25, 2018; Accepted: March 19, 2019; Published: May 6, 2019.

Reference information: J Clin Invest. 2019;129(6):2333-2350.

https://doi.org/10.1172/JCl123689. mechanism(s) underlying the existence of a window of opportunity after menopause remains unknown.

Memory extinction is proposed as a new form of learning (6), a notion that is supported by the observed properties of extinction as well as the fact that learning can be rapidly reacquired after extinction (7). It is also important to appreciate that extinction is not the same as forgetting (8). Forgetting implies that there is a decrease in the fear response due to the passage of time, whereas fear memories are actually resistant to forgetting, as they can persist for over a year (9). In addition, extinction is a fragile process, dependent on many factors, including timing relative to conditioning and stress (10). Neurotransmitters, including glutamate, serotonin, GABA, endocannabinoids (eCBs), and dopamine, play critical roles in the extinction phase (11). A role for eCBs in learning and memory, in fact, has been a focus for over a decade. Cannabinoid receptor 1-knockout (CB1-knockout) mice, for example, acquired conditioned and sensitized fear; however, knockout mice displayed impairments in extinction (12).

MicroRNAs (miRNAs) represent a class of small regulatory noncoding RNAs approximately $22 \mathrm{bp}$ in length, mediating post-transcriptional silencing of gene expression by recognizing specific sequences in target mRNAs (13). A single miRNA targets hundreds of different mRNAs, orchestrating epigenetic regulation of large combinations of gene products and facilitating developmental switches (14). miRNAs are highly expressed in the adult nervous system in a spatially and temporally controlled manner under normal physiologic as well as pathologic conditions (15). miRNAs have also been implicated in various aspects of dendrite remodeling and synaptic plasticity, as well as in experiencedependent adaptive changes of neural circuits during postnatal developmental and the adult brain (16). 17 $\beta$-Estradiol (E2) reg- 
ulates such miRNA transcription via estrogen receptors $\alpha$ and $\beta$ (ER $\alpha$ and $E R \beta$ ) in both a tissue-specific and a cell-dependent manner (17). E2-regulated miRNAs in normal homeostasis, reproduction, lactation, and cancer are therefore of great basic and clinical research interest (18). However, miRNA-mediated regulation with respect to an HT time window remains unclear.

In the present study, we found that $\mathrm{E} 2$ replacement improved fear memory formation in both $\mathrm{OVX}_{\mathrm{ST}}$ (1 week after OVX) mice and $\mathrm{OVX}_{\mathrm{LT}}(3$ months after OVX) mice but failed to promote fear extinction in $\mathrm{OVX}_{\mathrm{LT}}$ mice. High-throughput sequencing revealed a decrease of miR-221-5p in the medial prefrontal cortex (mPFC) of $\mathrm{OVX}_{\mathrm{LT}}$ mice. Decrease of miR-221-5p attenuated the levels of CB1 via Neurl1 $a / b$ mRNA and ubiquitin signaling. In addition, HT combined with a specific CB1 agonist rescued memory extinction in $\mathrm{OVX}_{\mathrm{LT}}$ mice. Our research uncovers a potential underlying mechanism with respect to an HT time window in long-term menopausal women and implies a new strategy for prolonging the window of opportunity for HT.

\section{Results}

Impaired fear memory extinction in E2-treated $O V X_{L T}$ mice. Previous research indicated that initiation of estrogen treatment at the time of menopause or soon after OVX provides a window of opportunity for the preservation of memory in females, whereas the administration of the hormone following a considerable delay in time after OVX exerts little or no beneficial effect on cognition (4). To verify this, we divided OVX mice into $\mathrm{OVX}_{\mathrm{ST}}$ and $\mathrm{OVX}_{\mathrm{LT}}$ mice, which received E2 replacement therapy 1 week or 3 months after OVX, respectively. E2 $(0.1 \mathrm{mg} / \mathrm{kg})$ was injected s.c. once daily for 1 week. Fear memory testing of mice was divided into memory formation phase and extinction phase. Normal mice usually perform more flexibility in different tasks, showing higher fear level in memory formation phase and lower fear level in extinction phase (19). Injection of $\mathrm{E} 2$ significantly promoted contextual fear memory in $\mathrm{OVX}_{\mathrm{ST}}$ and $\mathrm{OVX}_{\mathrm{LT}}$ mice and extinction in $\mathrm{OVX}_{\mathrm{ST}}$ mice; however, E2 impaired extinction in $\mathrm{OVX}_{\mathrm{LT}}$ mice (Figure 1A). Similar results were found in another kind of $\mathrm{MPFC}$-dependent trace fear memory (20) that required attention (Figure 1B), and working memory in a novel object recognition test (Figure 1C). Thus, E2 treatment did not rescue the performance of mice with $\mathrm{OVX}_{\mathrm{LT}}$ as it did in $\mathrm{OVX}_{\mathrm{ST}}$ $\mathrm{E} 2$ treatment ameliorated emotional disorders and motor learning impairment in $\mathrm{OVX}_{\mathrm{ST}}$ and $\mathrm{OVX}_{\mathrm{LT}}$ mice equally (Supplemental Figure 1; supplemental material available online with this article; https://doi.org/10.1172/JCI123689DS1). These results indicated that $\mathrm{E} 2$ replacement specifically impaired memory extinction and working memory in $\mathrm{OVX}_{\mathrm{LT}}$ mice.

E2 facilitates long-term potentiation, but not long-term depression, in $O V X_{L T}$ mice. The hippocampus, $\mathrm{mPFC}$, and amygdala are emerging as a circuit that participates in fear memory extinction (21). We therefore evaluated synaptic plasticity - long-term potentiation (LTP) and long-term depression (LTD) - in the hippocampus and $\mathrm{MPFC}$ in OVX mice with E2 treatment using a 64-channel multielectrode (MED64) system. Although E2 treatment facilitated the expression of theta burst stimulation-induced LTP in the hippocampus and MPFC of sham-operated, $\mathrm{OVX}_{\mathrm{ST}}$, and $\mathrm{OVX}_{\mathrm{LT}}$ mice, it only facilitated expression of low-frequency stimulation-induced LTD in sham and OVX mice, but not in $\mathrm{OVX}_{\mathrm{LT}}$ mice (Figure 2, A-F). Furthermore, E2 treat- ment prevented LTD in OVX $\mathrm{LT}_{\mathrm{LT}}$ mice. To identify the most reliable neuronal activation for memory extinction, we detected phosphorylated cFos ( $\mathrm{p}$-cFos) in the hippocampus, mPFC, and amygdala in sham mice. After 2 sessions of extinction training, p-cFos immunostaining indicated sustained $\mathrm{MPFC}$ activation compared with amygdala and hippocampus (Supplemental Figure 2). Thus, we thereafter focused our research on $\mathrm{mPFC}$, and analyzed dendritic spine density and morphology of mPFC neurons by Golgi-Cox staining. Spines are typically categorized into thin, filopodia, stubby, and mushroom according to their sizes and shapes (22). Mushroom and stubby spines are thought to be stable (containing the highest number of AMPA receptors) and appear to act as mature spines, while immature spines (thin and filopodia) are thinner protrusions with no clear head and harbor a small postsynaptic density (23). Although E2 treatment augmented both mature and immature spine density in $\mathrm{OVX}_{\mathrm{ST}}$ mice, it only increased mature spine density and failed to increase immature spine density in $\mathrm{OVX}_{\mathrm{LT}}$ mice (Figure 2, G and $\mathrm{H}$ ).

We asked whether alterations in estrogen and/or its receptors contributed to impaired fear extinction in $\mathrm{OVX}_{\mathrm{LT}}$ mice, but observed that levels of E1, E2, and E3 in plasma were similar between $\mathrm{OVX}_{\mathrm{ST}}$ and $\mathrm{OVX}_{\mathrm{LT}}$ mice after E2 treatment (Supplemental Figure 3A). The diminished levels of ER $\alpha$ in $\mathrm{OVX}_{\mathrm{ST}}$ and $\mathrm{OVX}_{\mathrm{LT}}$ mice were rescued by $\mathrm{E} 2$ treatment, while $\operatorname{ER} \beta$ did not change among treatments (Supplemental Figure 3B). Enzymes responsible for estrogen conversion were also not different among groups (Supplemental Figure 3B). These data therefore excluded the possibility that impairment of memory extinction in E2-treated OVX $\mathrm{LT}_{\mathrm{LT}}$ mice was due to $\mathrm{E} 2$ metabolism or receptor changes.

Fear extinction is closely related to LTD, and molecules such as glutamate, GABA, adenosine, and eCBs participate in the expression of $\operatorname{LTD}(19,24)$. Samples from the $\mathrm{mPFC}$ indicated that E2 treatment restored levels of GluN2A, GluN2B, and GluA1 in both $\mathrm{OVX}_{\mathrm{ST}}$ and $\mathrm{OVX}_{\mathrm{LT}}$ mice (Figure 2I). Interestingly, levels of $\mathrm{CB} 1$ receptors were changed in response to OVX. In OVX $\mathrm{ST}_{\mathrm{ST}}$ mice, levels of $\mathrm{CB} 1$ were increased but did not respond to E2 treatment, while levels of CB1 were decreased and were further reduced by E2 treatment in $\mathrm{OVX}_{\mathrm{LT}}$ mice. OVX induced alterations in metabotropic glutamate receptors (but this was independent of E2 treatment) but no changes of adenosine and GABA signaling among the groups (Supplemental Figure 4). The reduction of CB1 in the mPFC in $\mathrm{OVX}_{\mathrm{LT}}$ mice with E2 treatment was found in both the presynaptic and postsynaptic fractions (Supplemental Figure 5, A and B). Additionally, levels of CB1 were lower in the olfactory bulb, motor cortex, sensory cortex, caudate putamen striatum, and periaqueductal gray in $\mathrm{OVX}_{\mathrm{LT}}$ mice compared with $\mathrm{OVX}_{\mathrm{ST}}$ mice; however, levels of $\mathrm{CB} 2$ were similar between the groups (Supplemental Figure 5C).

Enhancing CB1 activity rescues memory extinction in E2-treated $O V X_{L T}$ mice. During the first 8 weeks of OVX, levels of CB1 decreased progressively in the $\mathrm{mPFC}$ (Figure 3A). Furthermore, levels of 2-arachidonoylglycerol (2-AG), one of the endocannabinoids (eCBs), were notably decreased in $\mathrm{OVX}_{\mathrm{LT}}$ mice and independent of $\mathrm{E} 2$ treatment; another eCB, anandamide (AEA), did not change in the mice (Supplemental Figure 6, A and B), although we found that levels of 2-AG were much higher than those of AEA in the mPFC. Levels of eCBs are determined by enzymes involved in the synthesis of 2-AG (DAGL $\alpha$ or DAGL $\beta$ ) and degradation of 2-AG (monoacylglycerol lipase [MGLL]) and AEA (FAAH) (25). Western blot data revealed 
A

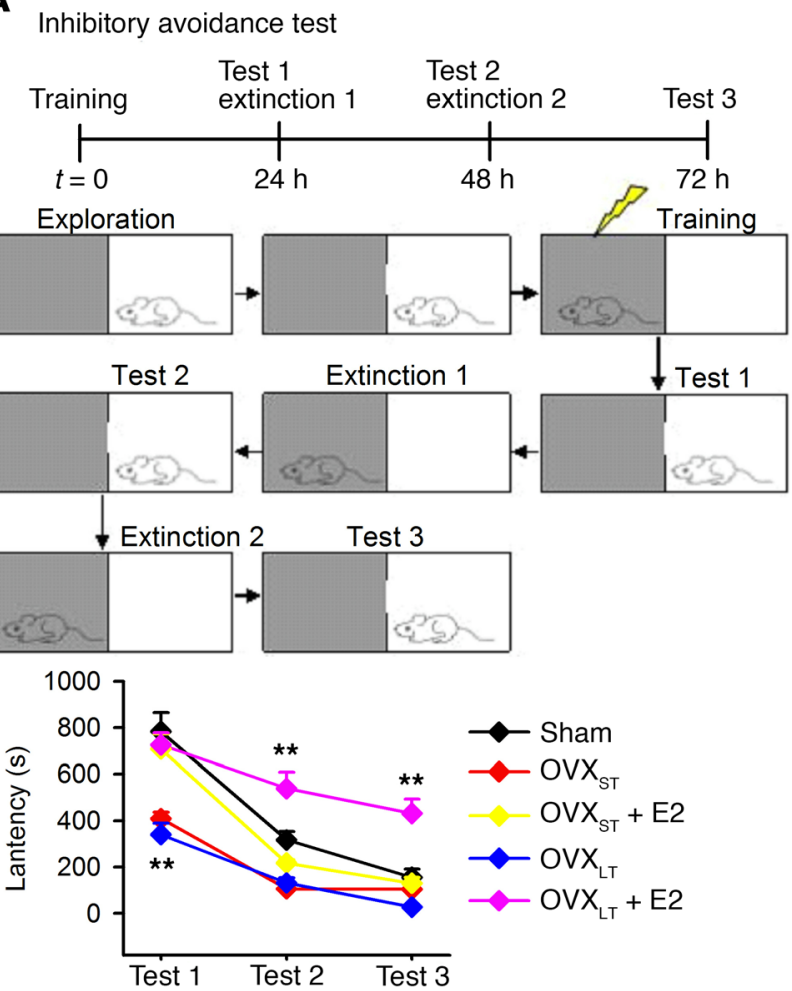

B

Trace fear memory test
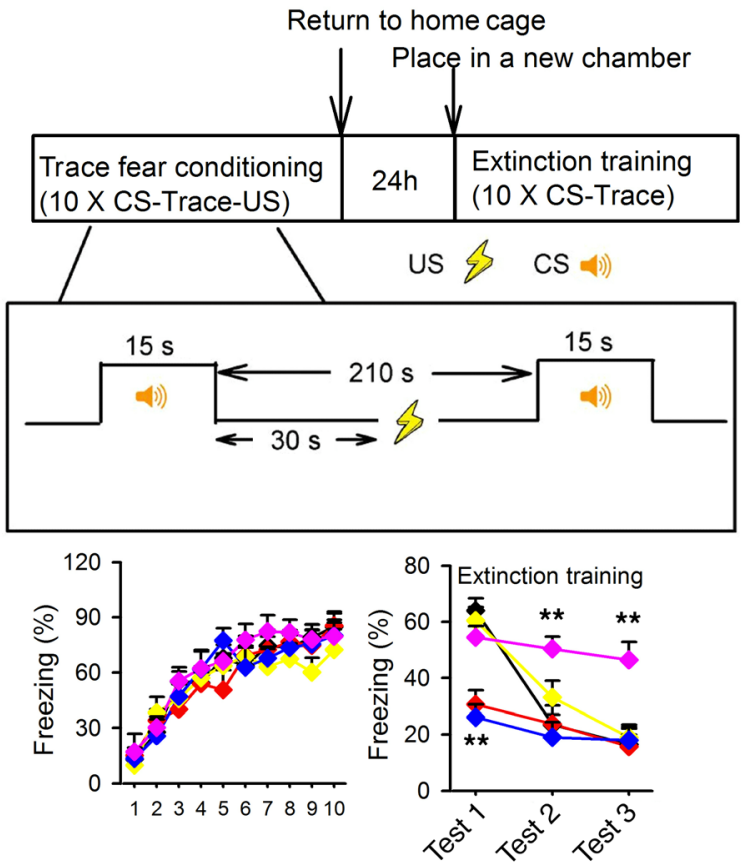

C
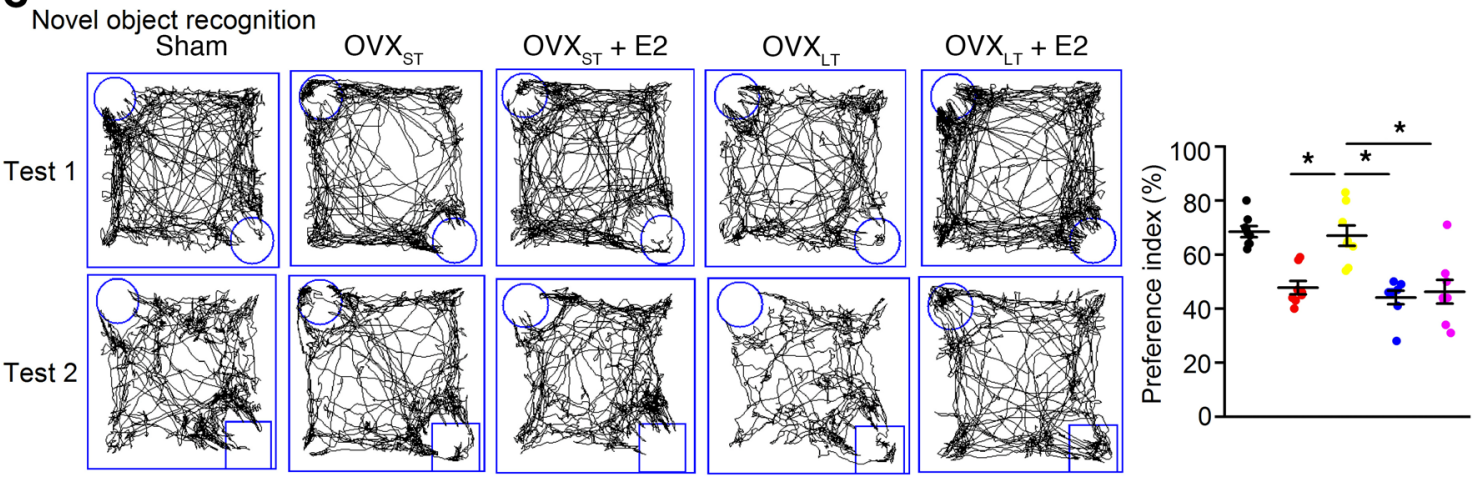

Figure 1. Impaired memory extinction in $\mathbf{O V X}$ mice treated with E2. (A) Schematic of inhibitory avoidance. Latency to dark room was recorded in sham, $\mathrm{OVX}_{S T}$, and $\mathrm{OVX}_{\mathrm{LT}}$ mice. (B) Schematic of trace fear memory. Freezing time was recorded in sham, $\mathrm{OVX}_{S T}$, and $\mathrm{OVX}_{\mathrm{LT}}$ mice. CS, conditioned stimuli (tone); US, unconditioned stimuli (electric shock). (C) Schematic of novel object recognition. Interaction time with novel and familiar objects was recorded in sham, $\mathrm{OVX}_{\mathrm{ST}}$, and $\mathrm{OVX}_{\mathrm{LT}}$ mice. Data are represented as mean $\pm \mathrm{SEM}, n=8$ mice per group. ${ }^{*} P<0.05 ;{ }^{*} P<0.01 \mathrm{vs}$. sham by 2 -way ANOVA followed by Bonferroni's post hoc test. Experimenters were blinded to the treatment.

lowered DAGL $\alpha$ expression in $\mathrm{OVX}_{\mathrm{LT}}$ mice, which may contribute to the lower 2-AG levels in the mPFC (Supplemental Figure 6, C and D).

Lower eCBs in the mPFC may lead to the impairment of synaptic transmission in the mPFC. Indeed, activation of CB1 by ACEA (1 $\mu \mathrm{M}$; a specific $\mathrm{CB} 1$ agonist) (26) induced a decrease in the field excitatory postsynaptic potential (fEPSP) slope in $\mathrm{mPFC}$ slices from sham and $\mathrm{OVX}_{\mathrm{ST}}$ mice, but not in $\mathrm{OVX}_{\mathrm{LT}}$ mice, indicating an insufficiency of eCBs in OVX $\mathrm{XT}_{\mathrm{LT}}$ mice (Figure 3B). Next, we explored whether activating $\mathrm{CB} 1$ receptors could restore memory extinction in $\mathrm{OVX}_{\mathrm{LT}}$ mice with E2 treatment. A combination of ACEA $(0.5 \mathrm{mg} / \mathrm{kg}$, s.c.) and E2 $(0.1 \mathrm{mg} / \mathrm{kg}$, s.c.) successively increased memory formation and restored memory extinction in $\mathrm{OVX}_{\mathrm{LT}}$ mice (Figure 3C), but did not affect the activities of $\mathrm{E} 2$ in pain sensation, motor activity, or anxi- ety- or depressive-like behaviors (Supplemental Figure 7). JZL184 (an MGLL inhibitor; $8 \mathrm{mg} / \mathrm{kg}$, s.c.) and WIN55,212-2 (a nonselective cannabinoid receptor agonist; $1 \mathrm{mg} / \mathrm{kg}$, s.c.) also rescued memory extinction impairment in $\mathrm{OVX}_{\mathrm{LT}}$ mice with $\mathrm{E} 2$ treatment. These effects were blocked by AM251 (a CB1 selective antagonist; $3 \mathrm{mg} / \mathrm{kg}$, s.c.) (Figure 3D), but not by AM630 (a CB2 selective antagonist; $1 \mathrm{mg} / \mathrm{kg}$, s.c.) (Supplemental Figure 7E). Interestingly, blocking CB1 with AM251 in E2-treated $\mathrm{OVX}_{\mathrm{ST}}$ mice also impaired extinction of fear memory (Supplemental Figure 7F). These results indicated a critical role for CB1 in memory extinction of $\mathrm{OVX}_{\mathrm{LT}}$ mice receiving $\mathrm{E} 2$ treatment.

To exclude off-target effects of chemicals, AAV-Cnr1-mCherry (which carried the CB1 gene virus) was locally injected into the mPFC of $\mathrm{OVX}_{\mathrm{LT}}$ mice, and AAV-mCherry alone was injected as 
A

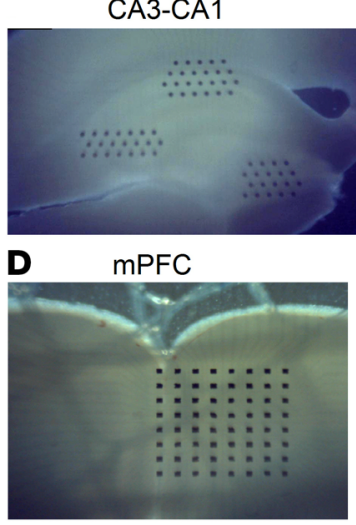

B

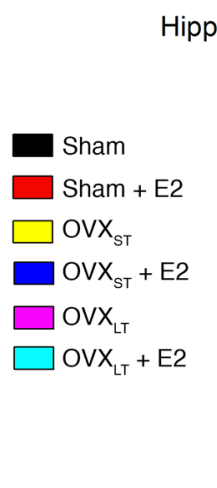

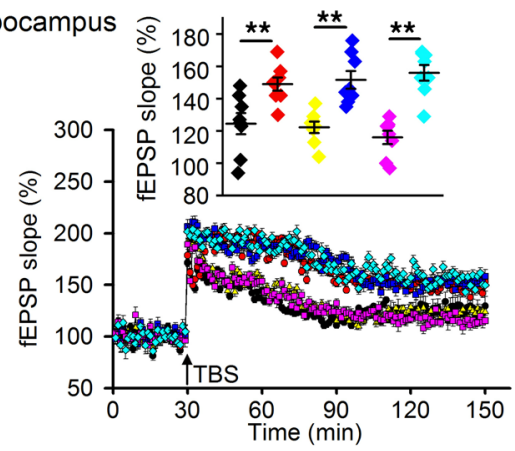

$\mathbf{F}$

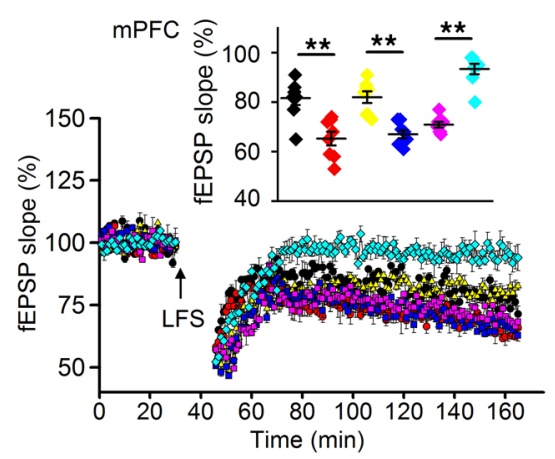

C
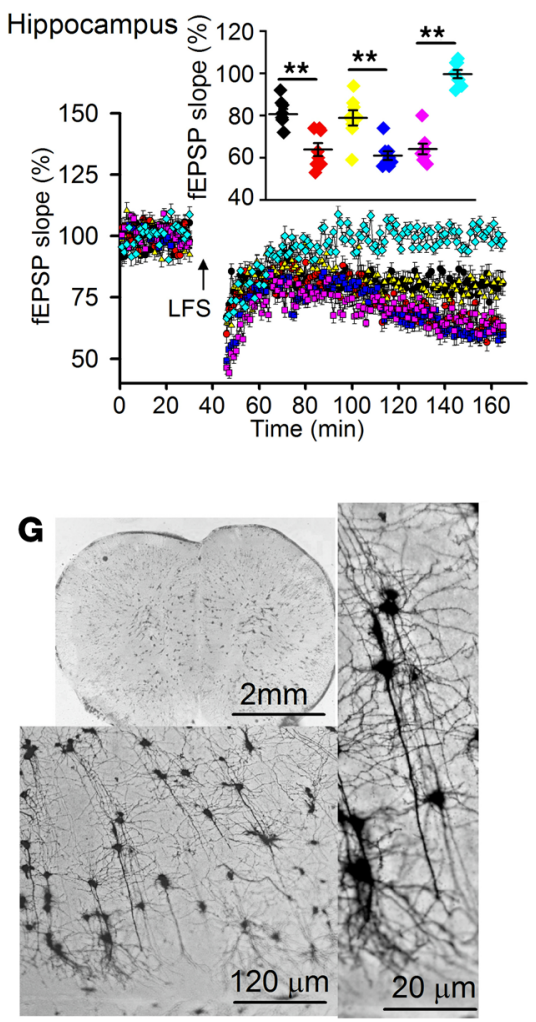

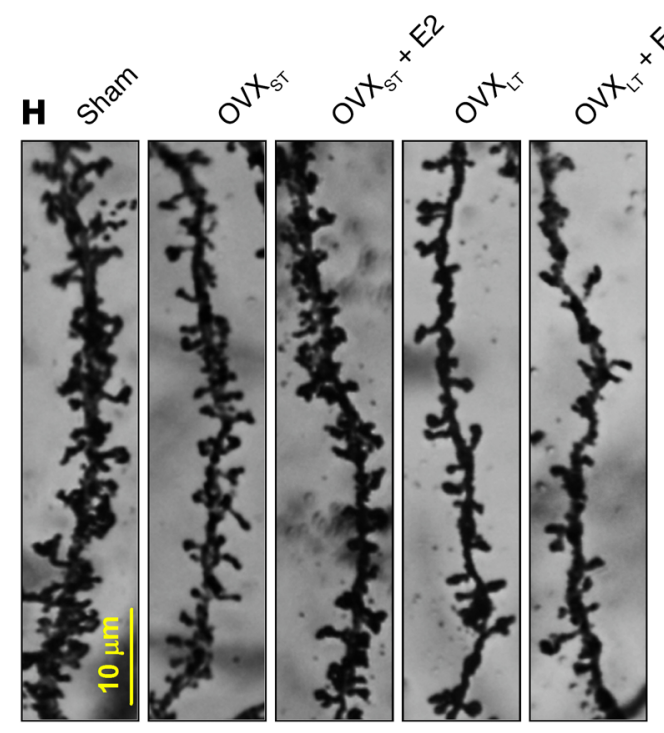

Sham $\square$ ovx $_{\mathrm{ST}} \square \mathrm{OVX}_{\mathrm{ST}}+\mathrm{E} 2$
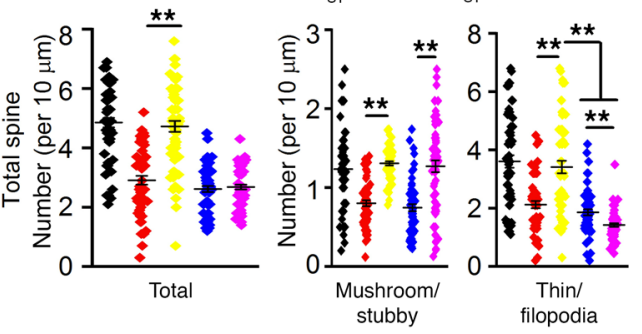

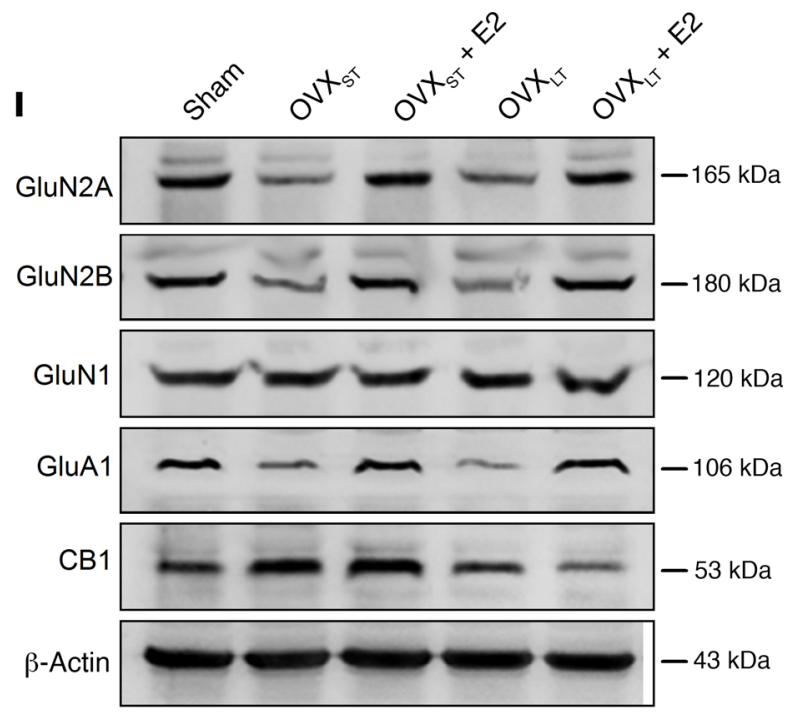

$\mathrm{OVX}_{\mathrm{LT}} \square \mathrm{OVX}_{\mathrm{LT}}+\mathrm{E} 2$

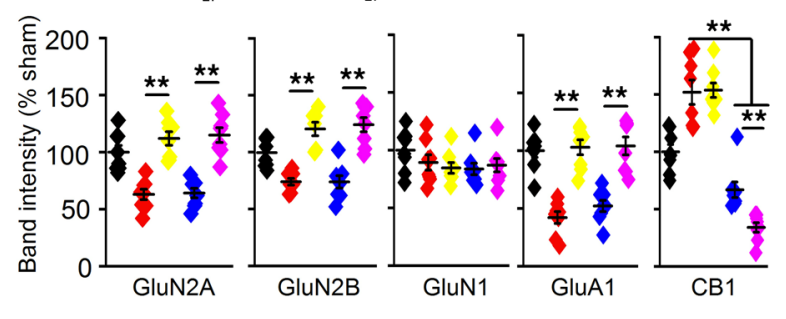


Figure 2. E2 failed to facilitate LTD and increase spine density in OVX mice. (A) Sample images showing the location of MED64 probe at CA1. (B and C) LTP and LTD recording in CA1 of hippocampus. Insets show accumulative field excitatory postsynaptic potential (fEPSP) slope at CA1 from different groups and averaged fEPSP slope during the last 30 minutes. Arrows indicate the time point of TBS or LFS application. $n=8$ slices from 4 mice per group. ${ }^{* *} P<0.01$ between the marked groups by 2-way ANOVA followed by Bonferroni's post hoc test. (D) Sample images showing the location of MED64 probe at MPFC. (E and F) LTP and LTD recording in MPFC. Insets show accumulative fEPSP slope at MPFC from different groups and averaged fEPSP slope during the last 30 minutes. Arrows indicate the time point of TBS or LFS application. $n=8$ slices from 4 mice per group. ${ }^{* *} P<$ 0.01 between the marked groups by 2-way ANOVA followed by Bonferroni's post hoc test. (G) Samples of Golgi-Cox staining of mPFC pyramidal neurons for spine counting. (H) Top: Representative images of basilar dendrites. Bottom left: Summary of total spine counts from basilar dendrites per 10 $\mu \mathrm{m}$. Bottom right: Number of spines including mushroom, stubby, thin, and filopodia types per $10 \mu \mathrm{m} . n=60$ neurons from 6 mice per group. ${ }^{* *} P<$ 0.01 between the marked groups by 2-way ANOVA followed by Bonferroni's post hoc test. (I) Top: Western blot samples showing the level of proteins in mPFC. Bottom: Summary of levels of CluN2A, GluN2B, GluN1, GluA1, and CB1 in mPFC. $n=8$ mice per group. ${ }^{* *} P<0.01$ between the marked groups by 2-way ANOVA followed by Bonferroni's post hoc test. Experimenters were blinded to the treatment. Data are represented as mean \pm SEM.

control (Figure 3E). CB1 overexpression with AAV-Cnr1-mCherry rescued fear extinction impairment (Figure 3F) and increased spine density of $\mathrm{mPFC}$ neurons in $\mathrm{OVX}_{\mathrm{LT}}$ mice given E2 (Figure $3 G)$. This increase was primarily due to "thin" spines, which are considered immature spines (27). These data further confirm the concept that $\mathrm{CB} 1$ is an important regulator of fear memory extinction in $\mathrm{OVX}_{\mathrm{LT}}$ mice.

E2 treatment causes a reduction in miR-221-5p in $O V X_{L T}$ mice. To elucidate the mechanism(s) underlying decreased CB1 in $\mathrm{OVX}_{\mathrm{LT}}$ mice, we performed high-throughput mRNA sequencing in mPFCs from different mice, but did not find any changes in CB1 mRNA levels among groups (Supplemental Figure 8). This lack of change in CB1 mRNA was confirmed by real-time PCR (Figure 4A), indicating a post-transcriptional mechanism underlying the reduction in CB1 receptor levels in $\mathrm{OVX}_{\mathrm{LT}}$ mice. We also observed an increase in prolactin mRNA (Supplemental Figure 8, C and D), but the prolactin antagonist bromocriptine $(3 \mathrm{mg} / \mathrm{kg}$, once daily for 1 week, i.p.) did not restore fear extinction in E2-treated $\mathrm{OVX}_{\mathrm{LT}}$ mice (Supplemental Figure 9).

Next, we performed miRNA sequencing in $\mathrm{MPFC}$ samples from different mice to screen potential target miRNAs (Supplemental Figure 10). Overlapping differently expressed genes in the $\mathrm{MPFC}$ were selected as potential targets (Figure 4B); and we thereby identified 2 decreased genes, miR-221-5p and miR-541-3p (Figure 4C), and confirmed these by quantitative real-time PCR (RT-PCR) (Figure $4 \mathrm{D})$. Then, we evaluated these same miRNAs in plasma samples from women aged 25-65, and found that miR-221-5p, but not miR$541-3 p$, was lower in women aged $56-65$, i.e., women who were approximately 10 years postmenopausal (Figure $4 \mathrm{E}$ ). Levels of 2-AG, total and free E2, and E3 were also lower in women aged 56-65 (Figure $4 \mathrm{~F}$ and Supplemental Figure 11). These data support our findings of decreased miR-221-5p and low 2-AG in $\mathrm{OVX}_{\mathrm{LT}}$ mice.

Role of miR-221-5p in LTD and fear extinction. To analyze the roles of miRNA in fear memory extinction and LTD expression, we treated mice with Cy3-tagged miR-221-5p agomir (0.5 nmol, 3 times per week i.c.v.) and Cy3-tagged miR-541-3p agomir (0.5 nmol, 3 times per week i.c.v.) (Figure 5A). Injection of miR-221-5p agomir, but not miR-541-3p agomir, rescued fear memory and LTD impairment in the MPFC of E2-treated $\mathrm{OVX}_{\mathrm{LT}}$ mice (Figure 5, B and C). Furthermore, miR-221-5p agomir increased CB1 levels in E2-treated $\mathrm{OVX}_{\mathrm{LT}}$ mice, and this effect was blocked by miR-221-5p antagomir (Figure 5D). Combination of E2 and miR-221-5p agomir facilitated fear memory extinction in inhibitory avoidance test and trace fear conditioning, but the addition of the CB1 agonist ACEA $(0.5 \mathrm{mg} / \mathrm{kg}$, s.c.) did not induce further facilitation (Figure $5 \mathrm{E}$ ). Interestingly, mPFC injection of miR-221-5p antagomir impaired fear memory extinction after $\mathrm{E} 2$ treatment in $\mathrm{OVX}_{\mathrm{ST}}$ mice, similar to that observed in E2-treated OVX $\mathrm{LT}_{\mathrm{LT}}$ mice (Figure $5 \mathrm{~F}$ ). These results indicated that lower levels of miR-221-5p constituted a critical driver of impaired fear memory extinction and LTD in E2-treated OVX ${ }_{\mathrm{LT}}$ mice.

miR-221-5p regulates ubiquitin-proteasome through Neurl1a/b. miRNA target prediction algorithms including TargetScan, PicTar, and miRanda did not predict CB1 as the miR-221-5p target, suggesting an indirect regulation of CB1 by miR-221-5p. To examine CB1 protein synthesis, SUnSET, a nonradioactive puromycin endlabeling assay, was used. This technique is used to measure protein synthesis during long-term associative memory consolidation (28). Equally facilitating new CB1 protein synthesis by E2 was found in sham, $\mathrm{OVX}_{\mathrm{ST}}$, and $\mathrm{OVX}_{\mathrm{LT}}$ mice (Supplemental Figure 12). These results excluded the possibility that the lower level of CB1 is due to decreased CB1 protein synthesis in $\mathrm{OVX}_{\mathrm{LT}}$ mice.

Using the DAVID functional annotation clustering tool, the Kyoto Encyclopedia of Genes and Genomes (KEGG), and Gene Ontology (GO), we predicted the genes linked to ubiquitin-mediated proteolysis as the miR-221-5p targets (Supplemental Figure 13A). Neurl1a and Neurl1b, 2 adaptor components of the E3 ubiquitin ligase complex (29-31), were thereby identified as potential targets (Figure 6A). Human HEK293 cells that expressed luciferase constructs containing Neurl1a and Neurl1b 3'-UTR-binding sites were used to determine the role of miR-221-5p in Neurl1a and Neurl1b expression. We observed that treatment of miR-221$5 p$ decreased luciferase activity in comparison with negative control cells (Figure 6B). Western blot analysis further verified that miR-221-5p depressed levels of Neurl1a and Neurl1b (Figure 6C), indicating that miR-221-5p regulates CB1 through the ubiquitinproteasome pathway. As predicted, CB1 levels were more sensitive to MG-132 (a 26S proteasomal inhibitor; $5 \mathrm{mg} / \mathrm{kg} / \mathrm{d}$, i.p., once daily for 1 week) in $\mathrm{OVX}_{\mathrm{LT}}$ mice relative to $\mathrm{OVX}_{\mathrm{ST}}$ mice (Figure 6D). The E2-induced CB1 decrease in $\mathrm{OVX}_{\mathrm{ST}}$ mice was notably reversed by MG-132 (Figure 6E). The autophagic-lysosomal pathway was not involved in CB1 degradation (Supplemental Figure 13B). Collectively, these data suggest that increased CB1 ubiquitination contributes to the lower levels of CB1 in E2-treated OVX $\mathrm{LT}_{\mathrm{LT}}$ mice.

CB1 degradation through Neurl1a/b-dependent ubiquitination. To confirm that the ubiquitin-proteasome pathway mediates CB1 degradation, we assessed the interaction between CB1 and ubiquitin, Neurl1a, or Neurl1b. As Figure 7A shows, CB1 directly interacted with ubiquitin, and Neurl1a or Neurl1b was increased in $\mathrm{OVX}_{\mathrm{LT}}$ mice compared with $\mathrm{OVX}_{\mathrm{ST}}$ mice. This binding was further enhanced after E2 treatment, implying that E2 enhanced CB1 ubiquitination in $\mathrm{OVX}_{\mathrm{LT}}$ mice. E2-induced phosphorylation of GluA1 at serine 831 and 845 was lost in $\mathrm{OVX}_{\mathrm{LT}}$ mice, but was 
A
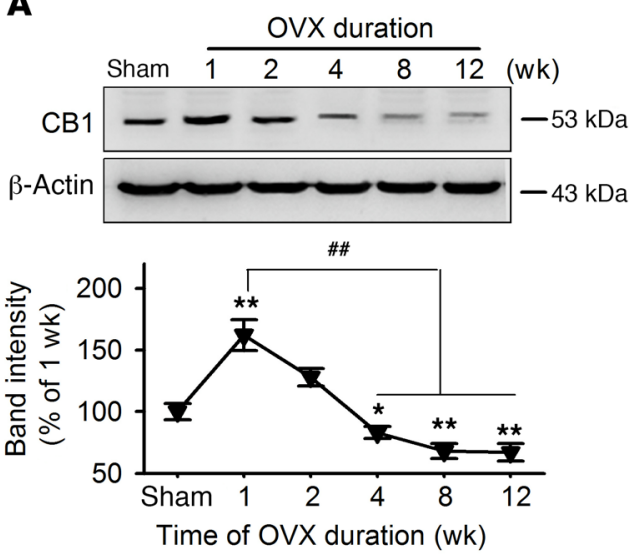

C

C Inhibitory avoidance test

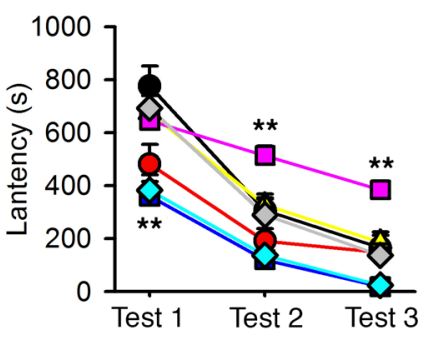

- Sham $-0-\mathrm{OVX}_{\mathrm{ST}}$ $\Delta \mathrm{OVX}_{\mathrm{ST}}+\mathrm{E} 2$ $\rightarrow-\mathrm{OVX}_{\mathrm{LT}}$ $\rightarrow-O V x_{L T}+E 2$ $\diamond \mathrm{OVX}_{\mathrm{LT}}+\mathrm{ACEA}$
B
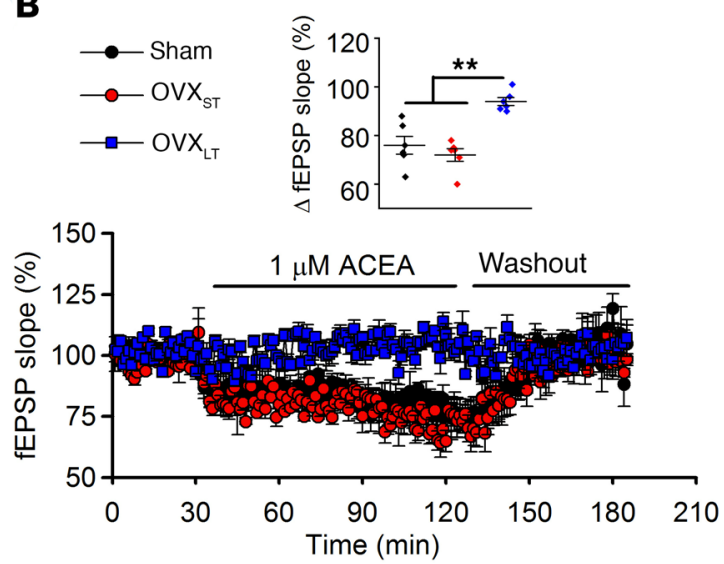

D

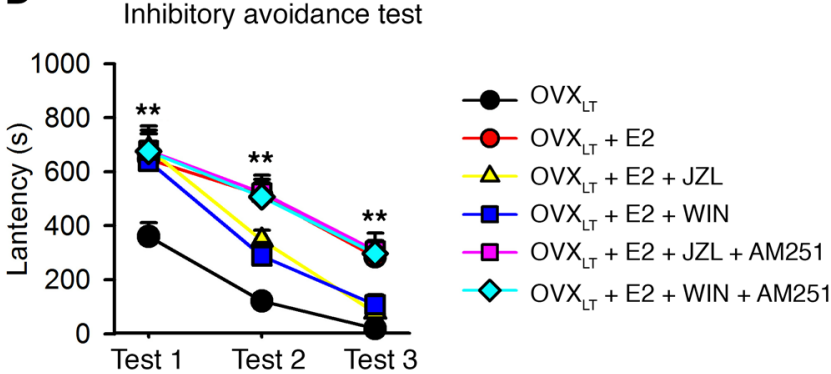

E $\diamond \mathrm{OVX}_{L T}+E 2+A C E A$

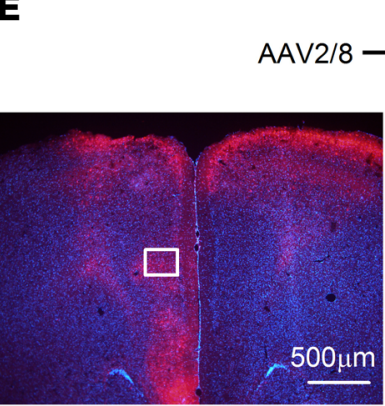
$\longrightarrow$ CMV mCherry DAPI Merged
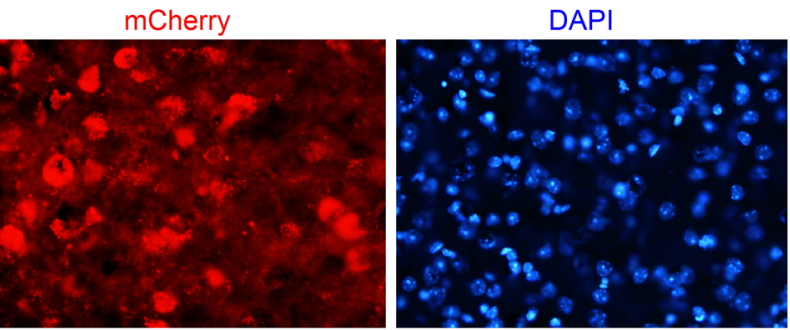

$\mathbf{F}$

OVX $L T$ mice

G inhibitory avoidance test
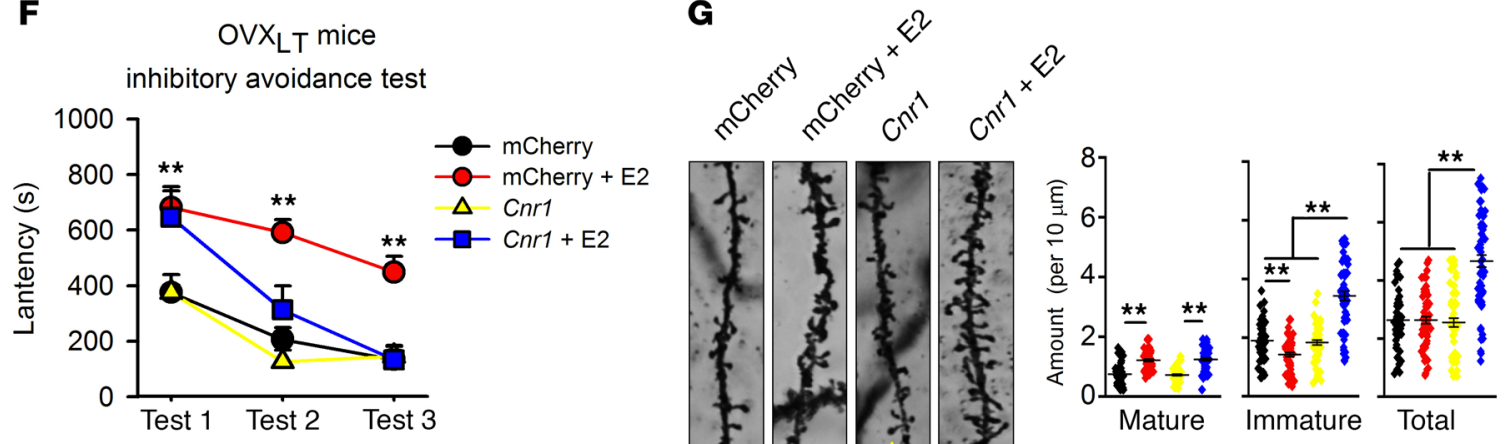
Figure 3. Decreased CB1 in $\mathrm{MPFC}$ and rescue of impairment of memory extinction by activation of CB1 in E2-treated $\mathrm{OVX}$ mice. (A) Levels of CB1 in mPFCs of mice after OVX. $n=6$ mice per group; 1-way ANOVA followed by Bonferroni's post hoc test; ${ }^{*} P<0.05$ and ${ }^{*} P<0.01$ vs. sham group, ${ }^{\#} P<0.01$ vs. mice 1 week after OVX. (B) Synaptic fEPSP response to ACEA (CB1 agonist, $1 \mu \mathrm{M}$ ) perfusion in mPFC slices from different mice. Inset shows the averaged fEPSP slope from 90 to 120 minutes. $n=8$ slices from 4 mice per group. ${ }^{* *} P<0.01$ between the marked groups by 1-way ANOVA followed by Bonferroni's post hoc test. (C) Effects of ACEA (0.5 $\mathrm{mg} / \mathrm{kg}$, s.c.) combined with E2 (0.1 mg/ $\mathrm{kg}$, s.c.) on fear memory formation and extinction. (D) Effects of JZL184 (2-AG hydrolysis enzyme inhibitor, 8 $\mathrm{mg} / \mathrm{kg}$, s.c.) or WIN55,212-2 (nonselective cannabinoid receptor agonist, 1 $\mathrm{mg} / \mathrm{kg}$, s.c.) combined with E2 on fear memory formation and extinction, and blockage of AM251 (CB1-specific antagonist, $3 \mathrm{mg} / \mathrm{kg}$, s.c.). (C and D) $n=8$ mice per group. ${ }^{* *} P<0.01 \mathrm{vs}$. sham or $\mathrm{OVX}_{L T}$ mice by univariate ANOVA after lower-bound correction as repeated-measurement data. (E) Images showing AAV-CMV-mCherry-Cnr1 injection in mPFC of OVX ${ }_{L T}$ mice. (F) Inhibitory avoidance test was carried out 4 weeks after AAV injection. $n=8$ mice per group. ${ }^{*} P<0.01$ vs. mCherry by univariate ANOVA after lower-bound correction as repeated-measurement data. (C) Representative images of basilar dendrites from mPFC neurons. Number of total and different spine subtypes. $n=60$ neurons from 6 mice per group. ${ }^{* *} P<0.01$ between the marked groups by 2-way ANOVA followed by Bonferroni's post hoc test. Experimenters were blinded to the treatment. Data are represented as mean \pm SEM.

maintained in $\mathrm{OVX}_{\mathrm{ST}}$ mice. This may be due to impaired synaptic transmission by long-term loss of CB1.

Local injection into the MPFC with AAV containing Neurl1a shRNA or Neurllb shRNA was used to knock down Neurl1a and Neurl1b levels (Figure 7B), and knockdown of Neurl1a or Neurl1b alone slightly increased CB1 levels while double knockdown increased CB1 dramatically. This suggests the existence of a compensatory relationship between Neurl1a and Neurl1b. miR-221-5p failed to regulate CB1 levels after knockdown of Neurl1a and Neurl1b (Figure 7C). As predicted, double knockdown of Neurl1a and Neurl1b rescued E2-induced fear extinction impairment in $\mathrm{OVX}_{\mathrm{IT}}$ mice (Figure 7D); and this effect was not enhanced by activation of CB1 by ACEA administration, suggesting that the same signaling pathway was targeted. The present data indicate that the miR-221-5p/Neurl1/ ubiquitin pathway mediates enhanced CB1 ubiquitination.

\section{Discussion}

In the present study, we identified the importance of CB1 in fear extinction impairment in long-term estrogen-deprived mice receiving estrogen treatment, and impaired LTD is known to be consistent with abnormal fear extinction. High-throughput RNA sequencing revealed an attenuated level of miR-221-5p, which targets the E3 ubiquitin protein ligases Neurl1a and Neurl1b. Higher E3 ubiquitin protein ligase activity, then, led to excessive CB1 ubiquitination, disrupting the $\mathrm{eCB}$ system in a retrograde fashion in long-term estrogen-deprived mice given estrogen (Figure 8). Thus, overexpression of miR-221-5p or synergistic activation of CB1 might prove to be potentially therapeutic in prolonging the HT time window in long-term menopausal women.

OVX mice are widely used as an animal model to study the human menopause and postmenopausal period. Mice are generally treated with $\mathrm{E} 2$ within 1 week (about 1 estrous cycle) after the OVX operation, while for women, the diagnosis of menopause is established by the absence of menstruation for 12 months (about
12 estrous cycles) or more (32), which is a relatively long time without cycles. Given the different lifespans between rodents and humans, deprivation of estrogen in mice for 3 months would be considered equivalent to long-term menopause in women. Some postmenopausal signs and symptoms in women, including cognitive deficit, anxiety, and depression, were found in present $\mathrm{OVX}_{\mathrm{LT}}$ mice. However, disparities between rodent and human findings may be deduced from an acute decline in E2 that is due to OVX and therefore distinct from natural menopause, where the loss of hormones is gradual and requires several years to complete. However, further biologic analyses revealed changes in estrogen levels, CB1, and miRNAs in the brains of OVX mice similar to those in human blood samples. Estrogen administration was beneficial but only if administered immediately after deprivation in the $\mathrm{OVX}_{\mathrm{sT}}$ mice, suggesting a so-called "time window of opportunity" in HT for OVX mice.

Natural menopause is a gradual process that occurs for most women between the ages of 47 and 55 (1). Observational studies found that individuals undergoing HT improved performance tests of working memory (33), verbal memory (34), and visual memory (35) compared with non-HT users. Findings from WHIMS and the Women's Health Initiative Study of Cognitive Aging (WHISCA) showed no cognitive advantages or decrease in risk for dementia in the HT groups $(36,37)$. The Kronos Early Estrogen Prevention Study (KEEPS) examined the effects of up to 4 years of HT on cognition and mood in recently postmenopausal women, aged 52.6, and 1.4 years after the last menstrual period. KEEPS, which randomized transdermal estradiol or conjugated equine estrogens versus placebo, showed no benefit to cognition (38). A study of younger women randomized to conjugated equine estrogen (with or without medroxyprogesterone) versus placebo between the ages of 50 and 55 by WHIMS, showed no benefit or risk to cognitive function measured at mean age of 67.2 (39). Additionally, the Early Versus Late Intervention Trial with Estradiol (ELITE) study showed no differential benefit of early (within 6 years) versus late (over 10 years) postmenopausal treatment (40). However, women who underwent surgical menopause earlier in life showed benefit of HT (41). Thus, HT is beneficial during a specific window of time (4) and the acute estrogen drop in surgical menopause. The inconsistency in the human literature could be explained by methodologic differences between studies, such as characteristics of populations enrolled, dose of drug, routes of administration, and methodologic issues, and these differences failed to convincingly explain discrepancies between studies in this area. In the present study, we verified a phenomenon like the "time window of opportunity" in a mouse model. E2 treatment notably improved fear memory formation and extinction shortly after OVX but had no effect on memory extinction in $\mathrm{OVX}_{\mathrm{LT}}$ mice (about 3 months after OVX). E2 treatment ameliorated emotional disorders and motor learning impairment equally in both $\mathrm{OVX}_{\mathrm{ST}}$ and $\mathrm{OVX}_{\mathrm{LT}}$ mice; however, it failed to rescue memory extinction and working memory in $\mathrm{OVX}_{\mathrm{LT}}$ mice. These data showed that only some menopausal symptoms can be ameliorated by HT after longterm estrogen deprivation, consistent with a putative critical time window for HT.

Most previous work regarding the role of $\mathrm{E} 2$ in cognition has focused on memory formation while ignoring memory extinction, 
A

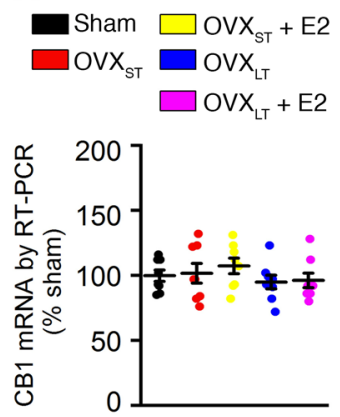

B

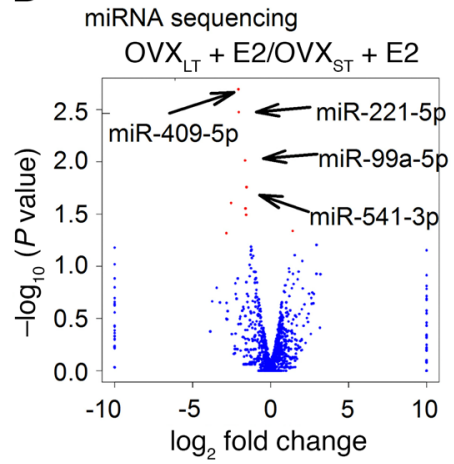

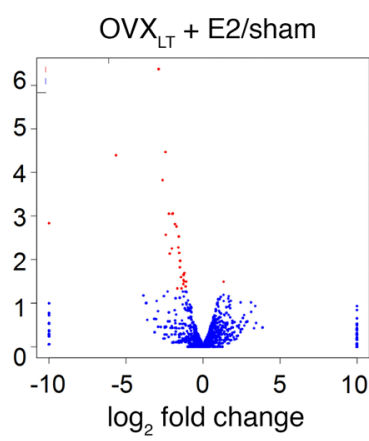

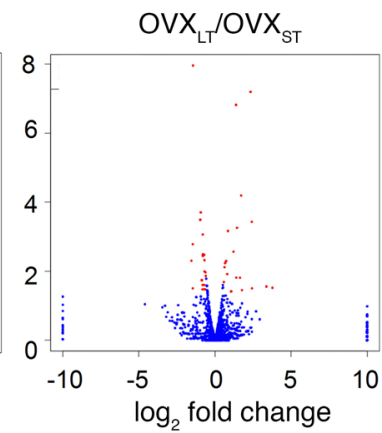

C

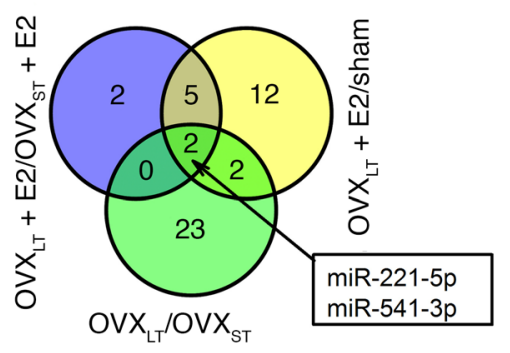

E

Plasma from women

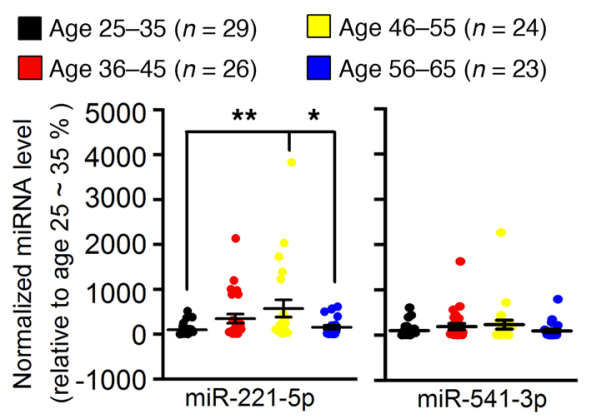

D

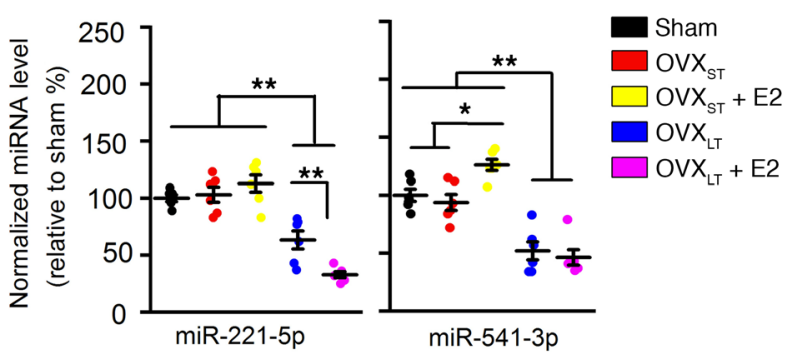
Duration of menopause

0-3 yr $(n=9)$ 4-7 yr $(n=9)$ $8-11$ yr $(n=10)$ $>12 \mathrm{yr}(n=6)$

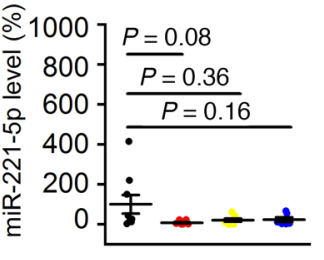

$\mathbf{F}$
Age 25-35 $(n=18)$

Age 36-45 $(n=18)$

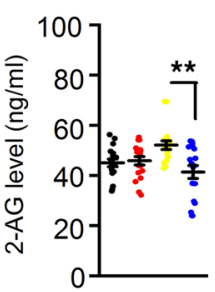

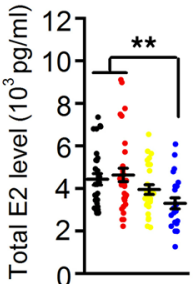

Age $46-55(n=18)$

Age $56-65(n=18)$

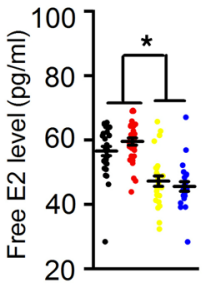

Figure 4. Decreased miR-221-5p after long-term estrogen deprivation. (A) mRNA levels of CB1 were similar among different mice. $n=8$ mice in each group. (B) Volcano plots of miRNA sequencing from the mPFC between $\mathrm{OVX}_{\mathrm{LT}}+\mathrm{E} 2$ and $\mathrm{OVX} \mathrm{ST}_{\mathrm{TT}}+\mathrm{E2} \mathrm{mice}_{\mathrm{OVX}}+\mathrm{OV}_{\mathrm{LT}}$ and sham mice, and $\mathrm{OVX} \mathrm{L}_{\mathrm{LT}}$ and $\mathrm{OVX} \mathrm{ST}_{\mathrm{ST}}$ mice. $n=3$ mice per group. (C) Venn diagram shows the potential miRNAs among treatments. miR-221-5p and miR-541-3p were recognized as targets responsible for impaired fear memory extinction in $\mathrm{OVX}_{\mathrm{LT}}+\mathrm{E} 2$ mice. (D) Levels of miR-221-5p and miR-541-3p in mPFC of different groups were confirmed by quantitative PCR. $n=6$ mice per group. ${ }^{*} P<0.05$, ${ }^{*} P<0.01$ between the marked groups by 2-way ANOVA followed by Bonferroni's post hoc test. (E) Levels of miR-221-5p and miR-541-3p in plasma from 25- to 35-year-old, 36- to 45-year-old, 46- to 55-year-old, and 56- to 65-year-old women. Right panel: Levels of miR-221-5p in plasma after years of menopause. (F) 2-AG (left), total E2 (middle), and free E2 (right) in plasma from different ages. (E and $\mathbf{F}){ }^{*} P<0.05,{ }^{*} P<0.01$ between the marked groups by 1-way ANOVA followed by Bonferroni's post hoc test. Experimenters were not blinded to the treatment. Data are represented as mean \pm SEM

but memory extinction is equally important for updating recognition, and is critical for the formation of new memories (42). Since synaptic plasticity is the cellular basis for learning and memory (43), LTP and LTD may play dominant roles in different phases of the aforementioned functions. LTP plays a critical role in the formation of Pavlovian fear-association learning, while LTD may be a molecular mechanism that facilitates the selective modification of a learned association to form a new memory (44). Fear extinction, rather, is a kind of selective modification of a learned association that keeps intact the ability to form new memories, which is in turn related to LTD (45). LTD is typically divided into NMDA receptordependent LTD and mGluR-dependent LTD (19). In both kinds of
LTD, eCBs can function as retrograde messengers that regulate synaptic plasticity in the striatum (46), neocortex (47), and cerebellum (48). Herein, we found that E2 facilitated LTP expression but resulted in failed expression of LTD in $\mathrm{OVX}_{\mathrm{LT}}$ mice. Chronic E2 treatment consistently improved fear memory formation, but this was disabled in the extinction phase in $\mathrm{OVX}_{\mathrm{LT}}$ mice. CB1, a vital molecule that mediates LTD, was lower in $\mathrm{OVX}_{\mathrm{LT}}$ mice compared with $\mathrm{OVX}_{\mathrm{ST}}$ mice. We found the increase of CB1 in the animals with short-term hormone deprivation. This result is consistent with findings of increased CB1 receptor density in OVX mice compared with cycling mice (49). 2-AG, an endogenous ligand of $\mathrm{CB} 1$ receptor, is decreased in $\mathrm{OVX}_{\mathrm{ST}}$ compared with sham mice. 
A
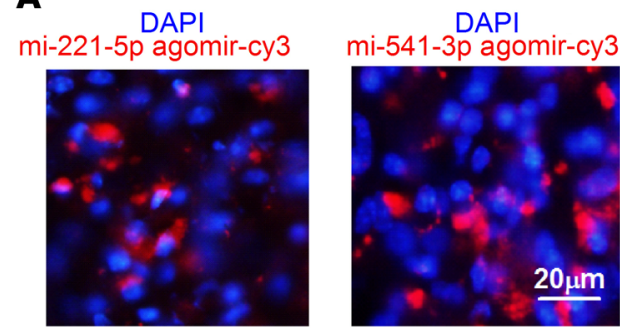

C

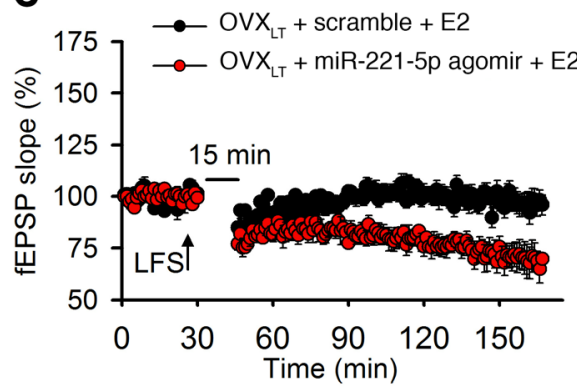

E

$$
\begin{aligned}
& \rightarrow \text { OVX }_{L T}+\text { scramble }+ \text { E2 } \\
& - \text { OVX }_{L T}+\text { miR-221-5p agomir }+ \text { E2 } \\
& \Delta \text { OVX }_{L T}+\text { miR-221-5p agomir + ACEA + E2 }
\end{aligned}
$$

Inhibitory avoidance test

Trace fear memory extinction
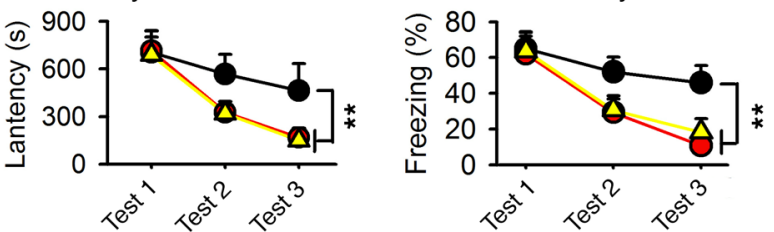

F

- $\mathrm{OVX}_{\mathrm{ST}}+$ scramble

-O- $\mathrm{OVX}_{\mathrm{ST}}+$ scramble $+\mathrm{E} 2$

$\Delta$ OVX $_{\mathrm{ST}}+\mathrm{miR}-221-5 \mathrm{p}$ antagomir

$\rightarrow-\mathrm{OVX}_{\mathrm{ST}}+\mathrm{miR}-221-5 \mathrm{p}$ antagomir + E2

Inhibitory avoidance test

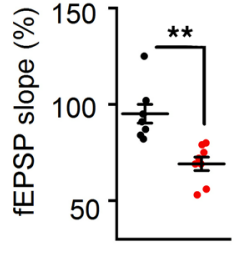

- $\mathrm{OVX}_{\mathrm{LT}}+$ scramble

- $-\mathrm{OVX}_{\mathrm{LT}}+$ scramble $+\mathrm{E} 2$

$\Delta \mathrm{OVX}_{\mathrm{LT}}+\mathrm{miR}-221-5 \mathrm{p}$ agomir

$\rightarrow-O V X_{L T}+m i R-221-5 p$ agomir $+E 2$

$-\mathrm{OVX}_{\mathrm{LT}}+\mathrm{miR}-541-3 p$ agomir

$\diamond \mathrm{OVX}_{\mathrm{LT}}+\mathrm{miR}-541-3 p$ agomir $+\mathrm{E} 2$

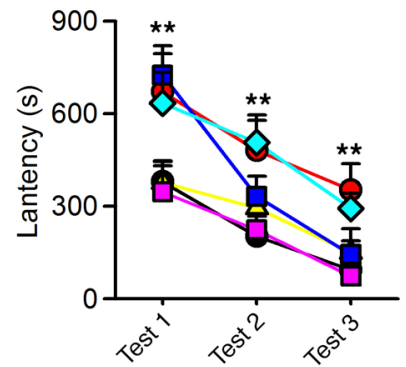

D
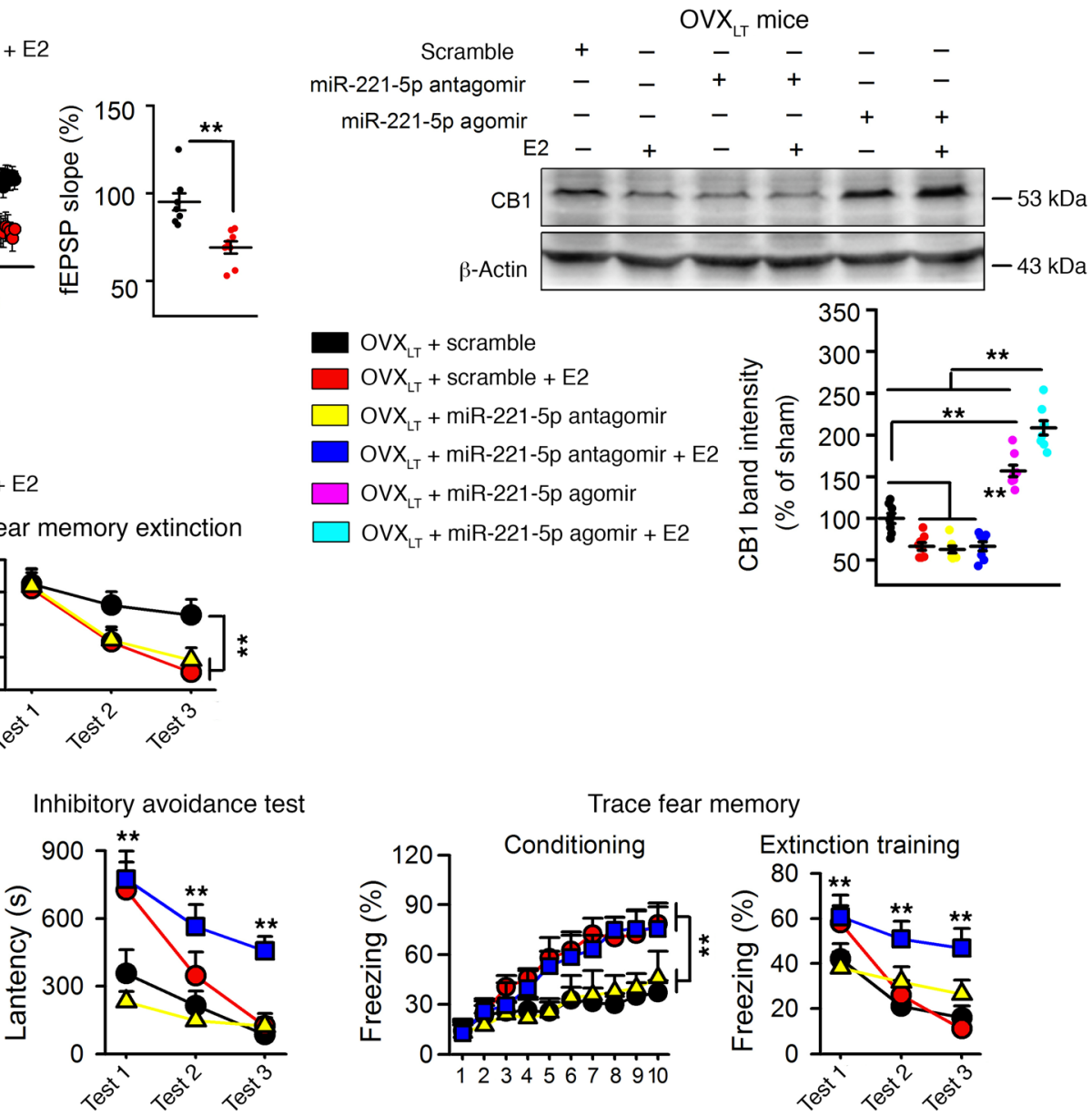

Figure 5. miR-221-5p responsible for impairment of fear extinction. (A) Sample images showing miR-221-5p agomir-Сy3 (red) and miR-541-3p agomir-Cy3 (red) injection and nucleus (DAPI, blue) in prefrontal cortex. (B) miR-221-5p agomir but not miR-541-3p agomir rescued memory extinction in E2-treated $\mathrm{OVX}_{\mathrm{LT}}$ mice. $n=8$ mice per group. ${ }^{* *} P<0.01 \mathrm{vs}$. scramble by univariate ANOVA after lower-bound correction as repeated-measurement data. (C) miR-221-5p agomir rescued impairment of LTD induced by E2 in $O V X_{L T}$ mice. Arrow indicates the point of LFS application. $n=8$ slices from 4 mice. ${ }^{* *} P<0.01$ by unpaired Student's $t$ test. (D) Increase of CB1 by miR-221-5p mPFC injection in E2-treated OVX mice. $n=5$ mice per group. ${ }^{* *} P<0.01$ between the marked groups by 2-way ANOVA followed by Bonferroni's post hoc test. (E) Combining miR-221-5p agomir with E2 + ACEA did not further increase memory extinction as compared with miR-221-5p + ACEA in $\mathrm{OVX}_{\mathrm{LT}}$ mice. $n=8$ mice per group. ${ }^{* *} \mathrm{P}<0.01$ between the marked groups by univariate ANOVA after lower-bound cor-

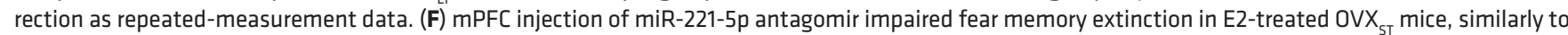
what was seen in E2-treated $\mathrm{OVX} \mathrm{L}_{L T}$ mice. $n=8$ mice per group. ${ }^{* *} P<0.01$ vs. scramble by univariate ANOVA after lower-bound correction as repeated-measurement data. Experimenters were blinded to the treatment except in C. Data are represented as mean \pm SEM.

Thus, the increase in $\mathrm{CB} 1$ expression in $\mathrm{OVX}_{\mathrm{ST}}$ mice may be a compensatory effect of decreased 2-AG. CB1-mediated depression of synaptic transmission was lost, indicating a dysfunction in $\mathrm{CB} 1$ receptors in $\mathrm{OVX}_{\mathrm{LT}}$ mice. ACEA combined with $\mathrm{E} 2$ treatment in $\mathrm{OVX}_{\mathrm{LT}}$ mice showed both normal fear memory formation and extinction. Other neurotransmitter receptors, including those for metabolic glutamate, GABA, and adenosine, were not involved in the modulation of extinction by E2. Our results, therefore, suggest the tantalizing possibility that $\mathrm{CB} 1$ is a candidate target to prolong the efficacy of E2 in treating late menopause.

Epigenetic changes are defined as mitotically and meiotically heritable changes in gene expression that do not involve a change 
A

SV40 Promoter Luciferase Neurl1a/b 3'-UTR poly(A)

(Neurl1a-1)

Position 838-844 of Neurl1a 3' UTR

5'.......CUACUGUUGCAGGGUUGCCAGGU..3

mmu-miR-221-5p

3' UGUCUUUAGAUGUAACAUACGGUCCA 5'

(Neurl1a-2)

Position 1508-1514 of Neurl1a 3' UTR

mmu-miR-221-5p

5' ......GGUGUUGAGAAUGGAUGCCAGGG..3'

|||\|||

3' UGUCUUUAGAUGUAACAUACGGUCCA 5'

(Neurl1b-1)

Position 1255-1261 of Neurl1b 3' UTR

mmu-miR-221-5p

(Neurl1b-2)

Position 1344-1351 of Neurl1b 3' UTR

5'.......UACUUGGAAGCCUGGUGCCAGGG.3'

| $|\|\||$ 3' UGUCUUUAGAUGUAACAUACGGUCCA 5

5' .......AAGCUUGAGACACCUUGCCAGGA 3'
mmu-miR-221-5p

C

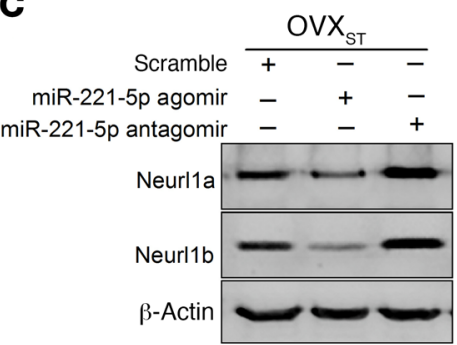

$\mathrm{OVX}_{\mathrm{ST}}$

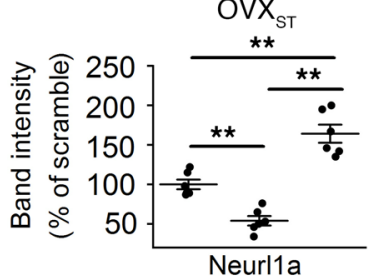

Neurl1a

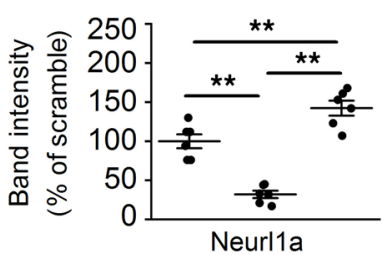

Scramble

\|\|\|\|

B

NC + WT 3'-UTR

$\square$ miR-221-5p + WT 3'-UTR

miR-221-5p + mutated 3'-UTR

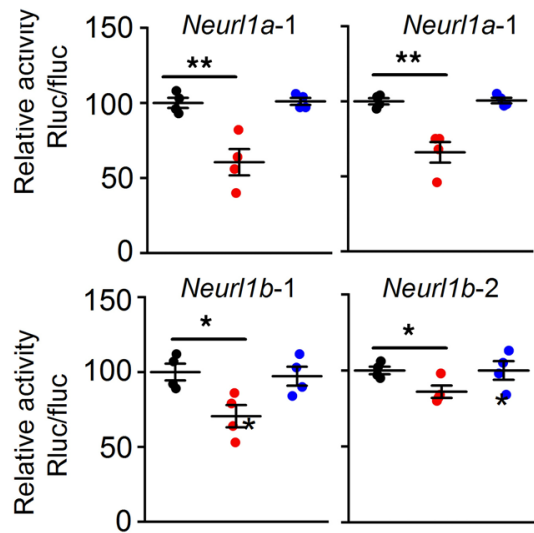

\section{3' UGUCUUUAGAUGUAACAUACGGUCCA 5'}
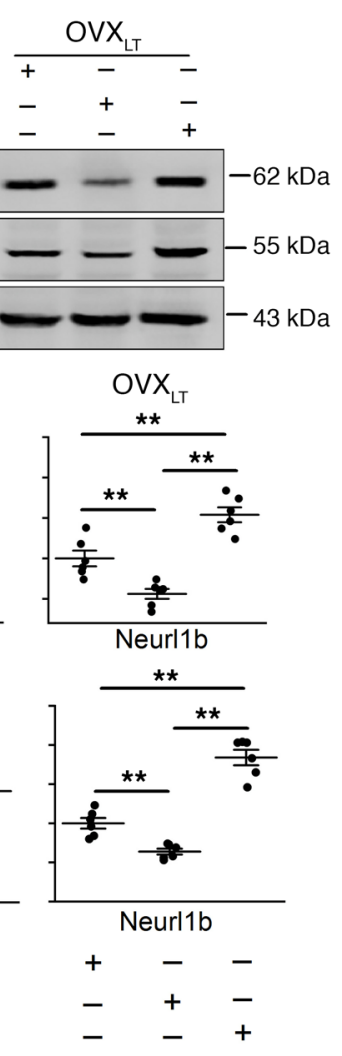

D
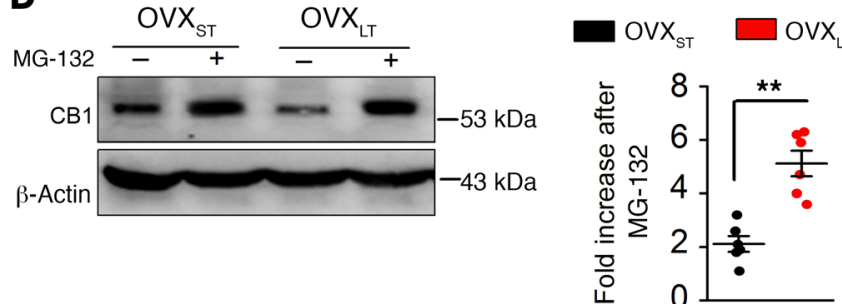

$\mathbf{E}$

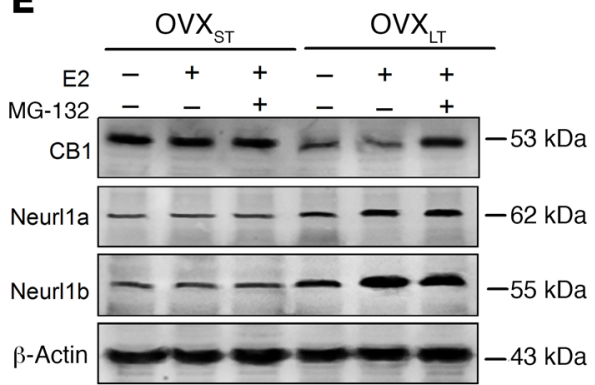

$$
\begin{array}{ll}
\square \operatorname{OVX}_{\mathrm{ST}} & \square \mathrm{OVX}_{\mathrm{LT}} \\
\mathrm{OVX}_{\mathrm{ST}}+\mathrm{E} 2 & \square \mathrm{OVX}_{\mathrm{LT}}
\end{array}
$$$$
\square \mathrm{OVX}_{\mathrm{ST}}+\mathrm{E} 2+\mathrm{MG}-132 \square \mathrm{OVX}_{\mathrm{LT}}+\mathrm{E} 2+\mathrm{MG}-132
$$
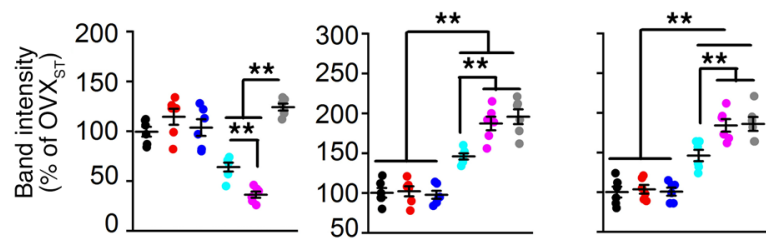

Figure 6. miR-221-5p regulating CB1 ubiquitination through Neurl1a/b-dependent pathway. (A) Alignment of binding sequences for miR-221-5p in the 3'-UTRs of Neurl1a and neurl1b mRNAs. (B) Interaction between miR-221-5p and 3'-UTRs of Neurl1a or neurl1b mRNAs detected by luciferase reporter gene. $n=4$ assays per group. ${ }^{*} P<0.05,{ }^{* *} P<0.01$ vs. negative control (NC) by 1-way ANOVA followed by Bonferroni's post hoc test. Rluc, renilla luciferase; Fluc, firefly luciferase. (C) mPFC injection of miR-221-5p agomir or antagomir regulated Neurl1a and Neurl1b expression in $\mathrm{OVX}_{\mathrm{ST}}$ and $\mathrm{OVX} \mathrm{X}_{\mathrm{LT}} \mathrm{mice} n=6 \mathrm{mice}$ per group. ${ }^{* *} P<0.01$ between the marked groups by 1-way ANOVA followed by Bonferroni's post hoc test. (D) MG-132 (a 265 proteasomal inhibitor, $5 \mathrm{mg} / \mathrm{kg}$, 1 week, once daily, i.p.) induced a larger increase of CB1 level in $\mathrm{OVX}_{\mathrm{LT}}$ mice than that in $\mathrm{OVX}_{\mathrm{ST}}$ mice. $n=6$ mice per group. ${ }^{* *} P<0.01$ by unpaired Student's $t$ test. (E) E2-induced decrease of CB1 was blocked by MG-132 in OVX ${ }_{L T}$ mice. E2 increased Neurl1a and Neurl1b levels in OVX $\mathrm{X}_{L T}$ mice, and higher Neurl1a and Neurl1 were found in $\mathrm{OVX}_{\mathrm{LT}}$ mice compared with $\mathrm{OVX}_{\mathrm{ST}}$ mice. $n=6$ mice per group. ${ }^{* *} P<0.01$ between the marked groups by 2 -way ANOVA followed by Bonferroni's post hoc test. Experimenters were not blinded to the treatment. Data are represented as mean \pm SEM. 
in DNA sequence. Epigenetic regulation plays a critical role in retaining long-term changes in postmitotic cells and regulates the formation and stabilization of long-term memory (50). miRNAs, as post-transcriptional epigenetic regulators, have been implicated in experience-dependent adaptive changes in neural circuits in the adult brain (16). To explore the underlying mechanism involved in lower $\mathrm{CB} 1$ levels in $\mathrm{OVX}_{\mathrm{LT}}$ mice, high-throughput miRNA sequencing was used and revealed diminutions in 2 miRNAs, miR221-5p and miR-541-3p. Combining this with the data from human samples, we considered miR-221-5p to be a critical regulator in women aged 56-65, i.e., women who were expected to be approximately 10 years postmenopausal. Luciferase assay indicated that miR-221-5p repressed Neurl1a and Neurl1b expression by binding to their 3'-UTRs. Upregulation of Neurl1a and Neurl1b by reduced miR-221-5p, then, increased the interaction between CB1 and ubiquitin, leading to $\mathrm{CB} 1$ degradation in $\mathrm{OVX}_{\mathrm{LT}}$ mice. Similar changes in miR-221-5p and CB1 were observed in women aged 56-65; concomitantly, levels of 2-AG and E2 were lower in women later in their postmenopausal period. miR-221-5p showed an agedependent increase from age 25 to age 55, indicating the developmental changes of miR-221-5p through unknown mechanisms. However, levels of miR-221-5p showed a dramatic decrease in women aged 56-65 compared with 46-55, which was quite consistent with the decrease of E2 during perimenopause. Our current findings therefore identify the importance of miRNA-mediated epigenetic changes that underlie the failure of E2 in improving cognitive performance in $\mathrm{OVX}_{\mathrm{LT}}$ mice.

The decrease of $\mathrm{CB} 1$ is more critical than the decrease of 2-AG in the impairment of memory extinction by $\mathrm{E} 2$ replacement in $\mathrm{OVX}_{\mathrm{LT}}$ mice. Another endocannabinoid, AEA, is normal in $\mathrm{OVX}_{\mathrm{LT}}$ mice and women aged 56-65. miR-221-5p replenishment restored CB1 level and thus rescued memory impairment in animals treated with miR-221-5p plus E2. Addition of the CB1 agonist ACEA did not induce further benefit; this could be explained by a simple floor effect.

There are several limitations in the present research. Firstly, mice with surgical but not natural menopause were used for standardizing menopause course, which could not mimic natural menopause in humans. Secondly, study of fear memory and LTP in mice could not fully represent the cognitive performance in humans. Thirdly, we could not exclude that other molecules might be medi-

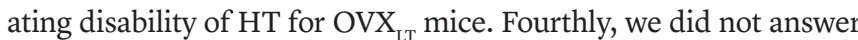
why miR221-5p was decreased in $\mathrm{OVX}_{\mathrm{LT}}$ mice and worsened after E2 replenishment. Further study needs to be done to prove miR221-5p/CB1-mediated failure of HT in postmenopausal women.

In summary, the present study provides evidence that $\mathrm{CB} 1$ is a potential target in the treatment of cognitive impairment in longterm menopause, and that CB1 agonist is a candidate for prolonging the HT time window.

\section{Methods}

Animals and drug treatments. Female C57BL/6J mice aged 12-15 weeks were used at the beginning of experiments. Mice were fed in groups of 3 per cage under standard laboratory conditions (12-hour light/12hour dark, temperature $22^{\circ} \mathrm{C}-26^{\circ} \mathrm{C}$, air humidity $55 \%-60 \%$ ) with water and mouse chow ad libitum. The experimental procedures were approved by the Institutional Animal Care and Use Committee of the
Fourth Military Medical University and complied with the Principles of Laboratory Animal Care (NIH publication 86-23, revised 1985). The number of animals used and their suffering were greatly minimized. Animals were ovariectomized (OVX) under pentobarbital sodium $(30 \mathrm{mg} / \mathrm{kg}$, i.p.). Briefly, a dorsal incision was made and ovaries were removed. Sham-operated controls were handled in the same manner as the OVX mice without removal of ovaries. E2 treatment was carried out by s.c. injection with $\mathrm{E} 2$ for 1 week $(0.1 \mathrm{mg} / \mathrm{kg}$, once daily) in olive oil. Drugs, except MG-132 and chloroquine, combining with E2 were mixed together in olive oil. MG-132 and chloroquine were diluted in saline and injected intraperitoneally. Equal volume of saline or olive oil was used as control. All behavioral data were collected by researchers blinded to the experiments.

Materials. All chemicals were purchased from Sigma-Aldrich unless stated otherwise. ACEA, WIN55,212-2, AM630, and JZL184 were purchased from Cayman Chemical. AM251, bromocriptine, MG-132, and chloroquine diphosphate were purchased from Abcam.

Inhibitory avoidance test. The inhibitory avoidance test, a singletrial aversive conditioning followed by learning and extinction sessions, was performed in shuttle boxes (San Diego Instruments) (51). All sessions started with a 90-second habituation to the compartments. Once the animal entered the dark compartment, the door was closed and the animal received a single electric foot shock of $0.5 \mathrm{~mA}$ intensity for 10 seconds. Experimental sessions included recording of latency to enter the dark compartment 24, 48, and 72 hours after conditioning. The 24and 48 -hour test sessions were followed by an extinction session during which animals were enclosed in the dark compartment for 200 seconds but did not receive a foot shock.

Trace fear memory and extinction. Trace fear conditioning training was performed using a Med Associates computer-controlled, sound-attenuated conditioning chamber $(29 \times 19 \times 25 \mathrm{~cm})$ as previously described (52). All data were recorded using a video-based FreezeFrame fear conditioning system and analyzed by Actimetrics Software (Coulbourn Instruments).

Novel object recognition. Novel object recognition test was performed as described previously (53). Mice were placed in the behavioral chamber to acclimate to the new environment (day 1). Each mouse was individually habituated to the apparatus for 10 minutes in the absence of objects (habituation trial). The next day (day 2), the mice were placed in the apparatus, and 2 identical cylinders were placed in a symmetrical corner of the box (training trial). On the test day (day 3), 1 cylinder was replaced with a cube of similar volume (testing trial). Time spent exploring each object during the training and test phase was scored by software (Shanghai Jiliang). Interaction parameters were defined as contact with the object (tail only excluded) or facing the object (distance $<2 \mathrm{~cm}$ ). The proportion of the exploration time exploring the novel object was defined as the "preference index" expressed by the ratio of $\mathrm{TN} /(\mathrm{TF}+\mathrm{TN})$, where $\mathrm{TF}$ is the time spent exploring the familiar object and $\mathrm{TN}$ is the time spent exploring the novel object.

Locomotor activity test. Locomotor activity test was conducted as described in a previous report (54). It was carried out in an open field, a square arena $(30 \times 30 \times 30 \mathrm{~cm})$ with clear Plexiglas walls and floor. Mice were placed in the center of the box and allowed to freely explore for 15 minutes. Mice were videotaped using a camera fixed above the floor and analyzed with a video tracking system.

Rotarod test. Motor learning was tested using an accelerating rotarod setup (Jiliang Software). Mice were habituated to a rotating 

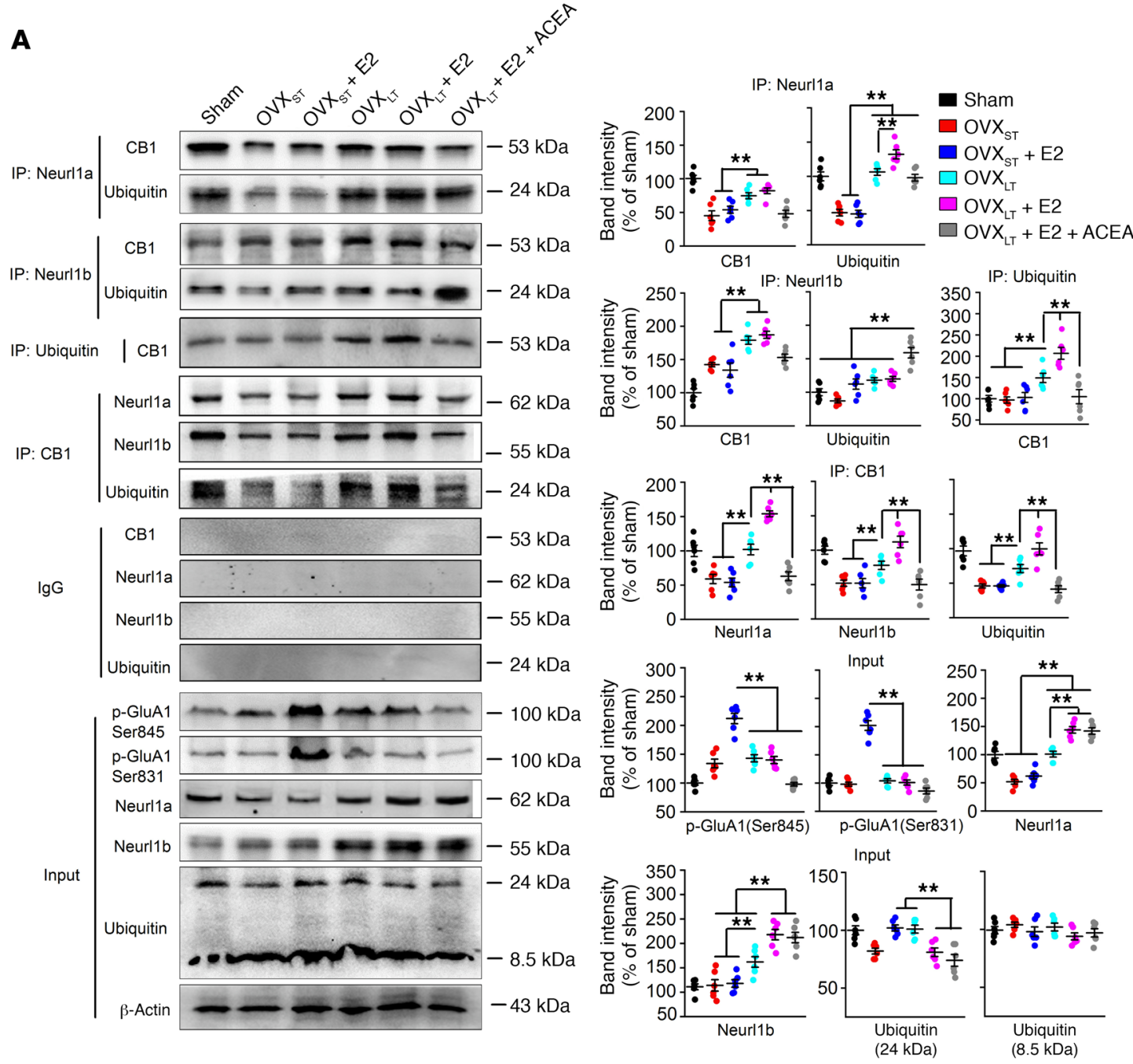

B

AAV-Neurl1a/b shRNA-GFP
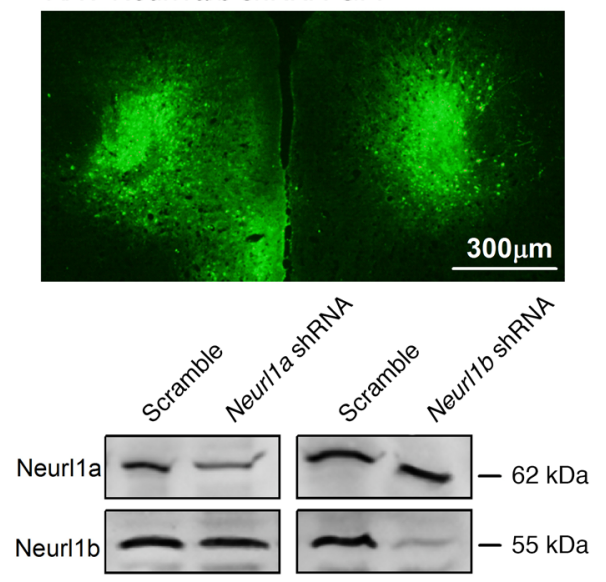

C

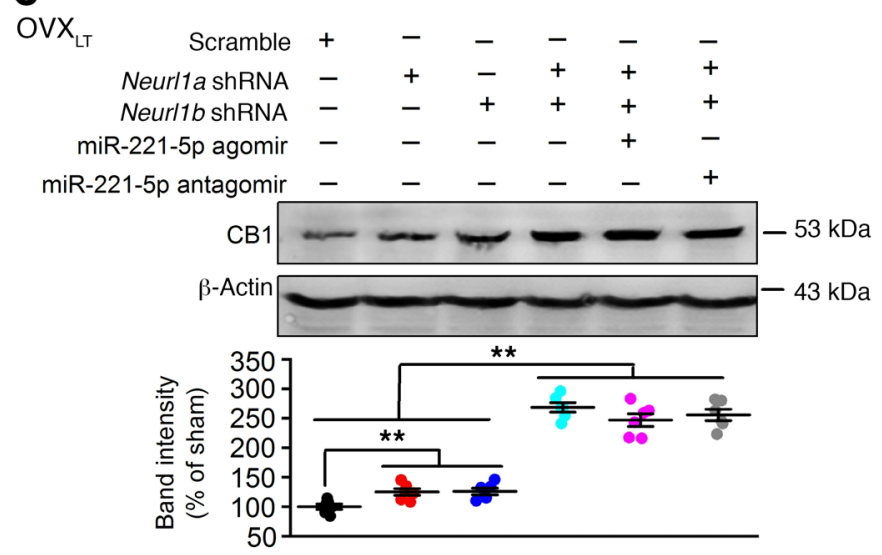

D

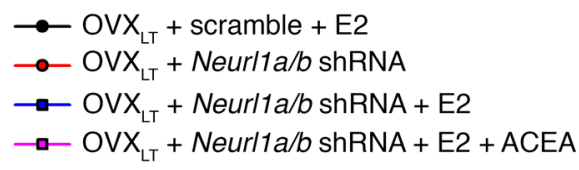

Inhibitory avoidance test

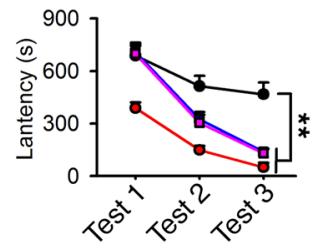

Trace fear memory extinction

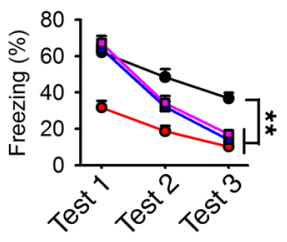


Figure 7. Increase of CB1 ubiquitination through Neurl1a/b-dependent pathway in $\mathbf{O V X}_{\mathrm{TT}}$ mice. (A) Coimmunoprecipitation showing the interaction between CB1 and ubiquitin, Neurl1a, or Neurl1b in MPFC and the effects of ACEA ( $0.5 \mathrm{mg} / \mathrm{kg}$, s.c.), E2 (0.1 mg/ $\mathrm{kg}$, s.c.), or miR-221$5 p$ agomir treatment on it. Ubiquitinated CB1 was increased after E2 treatment in $\mathrm{OVX}_{\mathrm{LT}}$ mice, which could be blocked by miR-221-5p agomir treatment. Phosphorylated GluA1 at Ser845 and Ser831 site was increased by $\mathrm{E} 2$ in $\mathrm{OVX}_{\mathrm{ST}}$ mice but not in $\mathrm{OVX}_{\mathrm{LT}}$ mice. $n=6$ mice per group. ${ }^{* *} P<0.01$ between the marked groups by 2-way ANOVA followed by Bonferroni's post hoc test. (B) Downregulation of Neurl7a and Neurl1b by mPFC injection of AAV-CMV-Neurl1a-shRNA-GFP and AAV-CMV-Neurl1b-shRNA-GFP in $O V X_{L T}$ mice. $n=6$ mice per group. (C) Levels of CB1 in $\mathrm{mPFC}$ of $O \mathrm{VX}_{\mathrm{LT}}$ mice after injection of Neurl1a shRNA, Neurl1b shRNA, miR-221-5p agomir, and/ or antagomir. $n=6$ mice per group. ${ }^{* *} P<0.01$ between the marked groups by 1-way ANOVA followed by Bonferroni's post hoc test. (D) Summary of latency in inhibitory avoidance test and freezing time in trace fear memory after extinction training in $\mathrm{OVX}_{\mathrm{LT}}$ mice injected with Neur/7a/b shRNA (mixture of Neurl1a shRNA and Neurl1b shRNA) or ACEA. $n=8$ mice per group. ${ }^{* *} P<0.01$ between the marked groups by univariate ANOVA after lower-bound correction as repeated-measurement data. Experimenters were not blinded to the treatment except in $\mathbf{D}$. Data are represented as mean \pm SEM.

drum at the slowest speed for 3 minutes before testing. Then, mice were tested on a rotating drum accelerated from 4 to $42 \mathrm{rpm}$ over a 5 -minute period. Time on the rod was measured before falling. Mice underwent 4 trials with an intertrial interval of 30 minutes. The longest time a mouse spent on the drum was analyzed.

Elevated plus maze. Elevated plus maze was conducted as described previously (55). The apparatus (DigBehv-EPMG, Jiliang Software) comprised 2 open arms $(25 \times 8 \times 0.5 \mathrm{~cm})$ and 2 closed arms $(25 \times 8 \times 12 \mathrm{~cm})$ that extended from a common central platform $(8 \times 8$ $\mathrm{cm}$ ) at a height of $50 \mathrm{~cm}$ above floor. Mice were videotaped using a camera fixed above the maze and analyzed with a video tracking system. Number of entries and time spent in each arm were recorded.

Forced swimming test. Swimming sessions were carried out by placement of mice in individual glass cylinders $(30 \mathrm{~cm}$ height $\times 10 \mathrm{~cm}$ diameter) containing water at $23^{\circ} \mathrm{C}-25^{\circ} \mathrm{C}, 25 \mathrm{~cm}$ deep, in such a way that mice could not support themselves by touching the bottom (56). Two swimming sessions were conducted: an initial 15-minute pretest (session inducing behavioral despair) followed 24 hours later by a 5 -minute test with only the last 4 minutes analyzed. After swimming, each mouse was dried, warmed, and returned to its home cage. Immobility time was recorded with a camera and analyzed by Actimetrics Software (Coulbourn Instruments).

Multichannel field potential recordings. Field potential recordings using a 64-channel recording system (MED64, Panasonic Alpha-Med Sciences) were described previously (57). One of 64 available planar microelectrodes located within the deep layer III of the mPFC or CA3 of hippocampus was selected as a stimulating electrode. Evoked field excitatory postsynaptic potentials (fEPSPs) were recorded from the other 63 channels. LTP was induced by a theta burst stimulation (TBS) protocol consisting of 5 trains of bursts with 4 pulses at $100 \mathrm{~Hz}$ and 200-millisecond intervals. TBS was repeated 5 times at intervals of 10 seconds. LTD was induced by low-frequency stimulation (LFS) (50-millisecond interstimulus interval) delivered at $1 \mathrm{~Hz}$ stimulation for 15 minutes (total pulses, 900). In each recording, 6-8 channels were selected for analysis. To test E2 effects on plasticity, slices were preincubated in $1 \mathrm{nM}$ E2 for 1-2 hours, and E2 was in the artificial cerebrospinal fluid during the whole recording session. ACEA $(1 \mu \mathrm{M})$ was added at indicated time points and washed out. LTP and LTD strengths were estimated by fEPSP slope 90 minutes after TBS or LFS stimulus. Effects of ACEA were estimated from 60 minutes after drug administration.

Golgi staining and spine morphology analysis. Golgi staining was performed as described previously (58). Briefly, unfixed whole brains were carefully removed and then immersed in $10 \mathrm{ml}$ of Golgi-Cox solution (1\% potassium dichromate, $1 \%$ mercuric chloride, and $0.75 \%$ potassium chromate) and stored in a tightly sealed glass jar in the dark at room temperature for 12 days. After dehydration in $70 \%, 80 \%$, and $100 \%$ ethanol and ethanol/diethyl ether (1:1), brains were sectioned $(120 \mu \mathrm{m})$ using a vibratome in distilled water. After washing in distilled water for 5 minutes, the sections were developed in a solution of $16 \%$ ammonium hydroxide for 30 minutes and rinsed using distilled water for another 5 minutes. The sections were immersed in 1\% sodium thiosulfate to fix the stain for 10 minutes at room temperature, and then washed 3 times for 5 minutes each in distilled water. Each slide was coverslipped using Permount and imaged under an Olympus BX51 microscope with a $\times 100 /$ NA 1.4 oil immersion lens. Basal dendrites were randomly selected for imaging. Dendritic spine density was calculated as number of spines per $10 \mu \mathrm{m}$ dendritic length from the soma using Image (NIH). Spine density of basal dendrites was obtained in $10-\mu \mathrm{m}$ segments starting $25 \mu \mathrm{m}$ from the soma along the entire length of basal dendrite (in all cases, at least $100 \mu \mathrm{m}$ ). Ten neurons from 5 sections were analyzed for each mouse. Images of spine visible along the entire length of basal dendrite (in all cases, at least $100 \mu \mathrm{m}$ ) were taken. We did not count the spines by $Z$-stacks of the images or 3-dimensionally. Spines less than about $0.4 \mu \mathrm{m}$ in profile length were not counted; these spines were usually those projecting at oblique angles and partially obscured by overlap with the dendritic shaft. Spine morphology was classified according to the criteria: "thin" type with a long neck and small head; "stubby" type with a large head but no neck; "mushroom" type with a large head and thick neck. Values from neurons derived from the same animal were averaged. All image analyses and processing were carried out in a blinded manner using Image $(\mathrm{NIH})$.

RNA purification from $M P F C$. Mice were anesthetized using isoflurane and decapitated by guillotine. MPFCs were quickly isolated on ice-cold glass slides, placed in frozen vials containing RNA Stabilization Reagent (QIAGEN) at $4^{\circ} \mathrm{C}$, and stored at $-80^{\circ} \mathrm{C}$ for subsequent analyses. Total RNAs from mPFC were isolated with TRIzol Reagent (Life Technologies) based on the manufacturer's instructions. RNA samples stored in dried ice were delivered to Sangon Biotech for mRNA and miRNA sequencing analysis. Concentrations of total RNA, RNA integrity number (RIN), and ratio of $28 \mathrm{~S}$ to $18 \mathrm{~S}$ ribosomal RNA were measured. Samples were selected for the construction of transcriptome and small RNA libraries with total RNA amount over $10 \mu \mathrm{g}$, concentration over $200 \mathrm{ng} / \mu \mathrm{l}$, RIN over 8, and ratio of $28 \mathrm{~S}$ to $18 \mathrm{~S}$ over 1.0.

$m R N A$ and miRNA sequencing. RNA sequencing was conducted by Sangon Biotech. Briefly, TruSeq RNA Sample Prep kit (Illumina) and $1 \mu \mathrm{g}$ of total RNA were used to make poly(A)-selected and barcoded RNA sequencing libraries for each $\mathrm{mPFC}$ sample. Adaptors containing 7 nucleotide indexes were ligated to the double-stranded cDNA. DNA was purified between enzymatic reactions, and size selection of the library was performed with AMPure XT beads (Beckman Coulter Genomics). Approximately 17- to 27-nt-long RNA fragments (enriched 


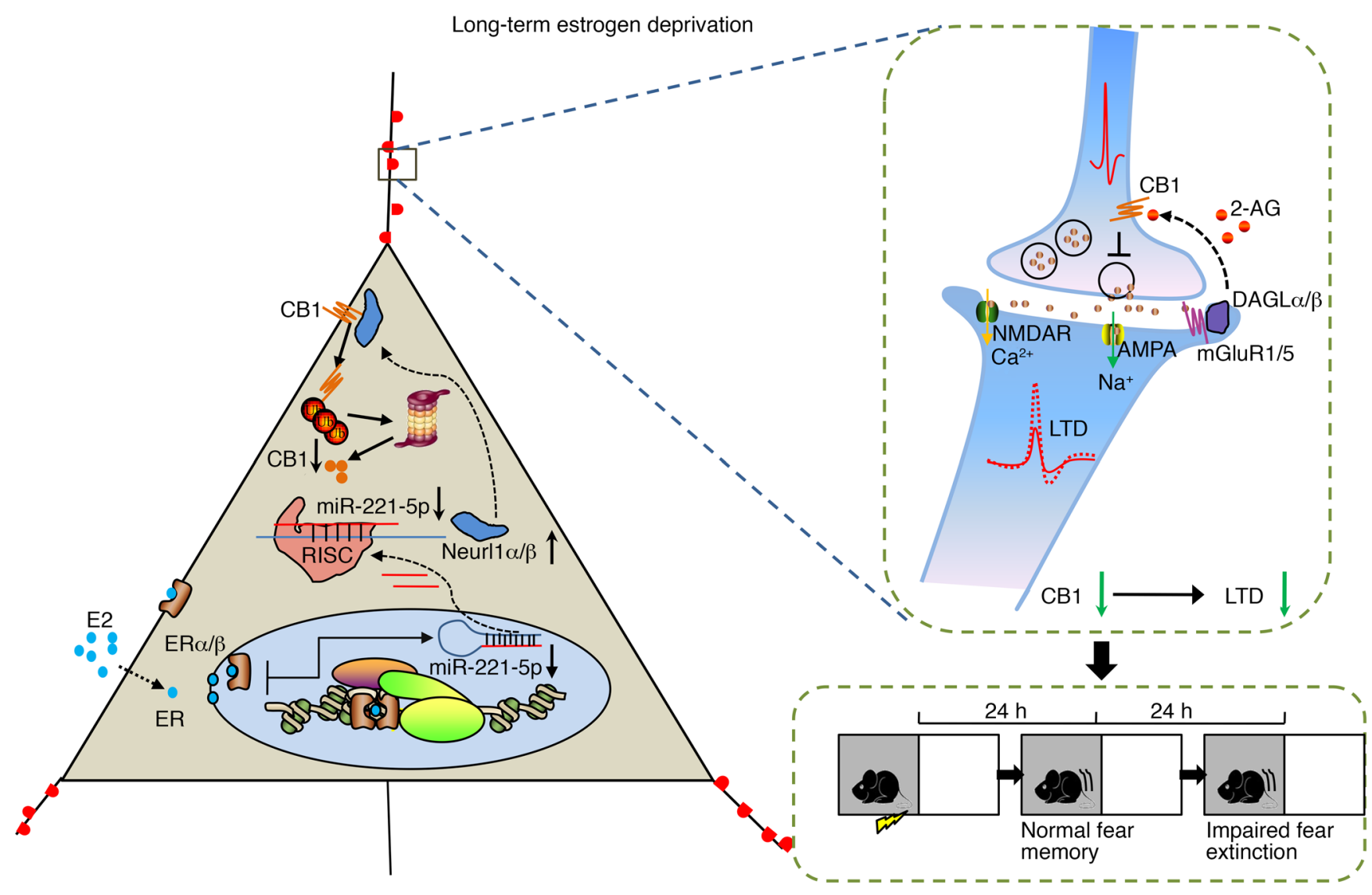

Figure 8. Schematic of E2/CB1-mediated signaling in long-term estrogen deprivation. Left: In long-term estrogen-deprived mice, E2 treatment activates downstream effector molecules. It leads to reduction of miR-221-5p, which targets the E3 ubiquitin protein ligases Neurl1a and Neurl1b. Higher E3 ubiquitin protein ligase activity, then, leads to excessive CB1 ubiquitination. Right: CB1 is a retrograde receptor and critical for induction of LTD. Reduction of CB1 leads to abnormal LTD induction and fear extinction in long-term estrogen deprivation. RISC, RNA-induced silencing complex.

for miRNAs) were isolated from the total RNAs using denaturing PAGE (15\%). Deep-sequencing libraries were constructed using Small RNA Sample Prep kit (Illumina). $3^{\prime}$ and $5^{\prime}$ adaptors were ligated to purified miRNAs sequentially. Adaptor-tagged miRNAs were reversetranscribed and amplified by low-cycle PCR. PCR products were purified by PAGE (8\%) and sequenced using a Genome Analyzer II (Illumina). Libraries were assessed for concentration and fragment size by DNA High Sensitivity Assay on the LabChip GX (PerkinElmer) and quantitative PCR using the KAPA Library Quantification Kit (Complete, Universal; Kapa Biosystems). Libraries were pooled and sequenced on a 100-bp paired-end Illumina HiSeq 2500 run (Illumina). Sequenced reads were aligned to the reference sequence (Ensembl version Rnor5.0.77) using TopHat version 1.4.1 (59). The alignments allowed up to 2-bp mismatches per 25-bp segment. Measurement of transcript abundance and identification of splice variants were done by Cufflinks version 1.3 using the BAM alignment files obtained from TopHat (60). BigWig coverage files were generated from the BAM alignment files using the UCSC genome browser tools. Target gene levels were predicted by miRDB database. Identified genes were subjected to functional annotation, clustering, and analysis using KEGG analysis based on the Database for Annotation, Visualization, and Integrated Discovery (DAVID) bioinformatics database (https://david.ncifcrf.gov/). The mRNA and miRNA sequencing data were uploaded to the NCBI's Sequence Read Archive (SRA) database (SRA accession PRJNA524977).
miRNA agomir and antagomir injection. The sequences of miRNA agomirs and antagomirs were as follows: scramble agomir: 2'-O-methylated-sulfo-5'-Cy3-UUCUCCGAACGUGUCACGUTTcholesterol-3' (sense), 2'-O-methylated-sulfo-5'-Cy3-ACGUGACACGUUCGGAGAATT-cholesterol-3' (antisense); scramble antagomir: 2'-O-methylated-sulfo- $5^{\prime}$-Cy3-UUGUACUACACAAAAGUACUGcholesterol-3'; miR-221-5p agomir: 2'-O-methylated-sulfo-5'-Cy3-ACCUGGCAUACAAUGUAGAUUUCUGU-cholesterol-3' (sense), 2'-Omethylated-sulfo - 5 ' - Cy 3-AGAAAUCUACAUUGUAUGCCAGGAUU-cholesterol-3' (antisense); miR-221-5p antagomir: 2'-O-methylated-sulfo-5'-Cy3-ACAGAAAUCUACAUUGUAUGCCAGGU-cholesterol-3'; miR-541-3p agomir: 2'-O-methylated- sulfo5'-Cy3-UGGCGAACACAGAAUCCAUACU-cholesterol-3' (sense), 2' -O-methylated-sulfo-5' - Cy3-UAUGGAUUCUGUGUUCGCCAUU-cholesterol-3' (antisense); miR-541-3p antagomir: 2'-O-methylated-sulfo-5'-Cy3-AGUAUGGAUUCUGUGUUCGCCA-cholesterol-3'.

Oligoribonucleotides were injected as described previously (61, 62). Agomir, antagomir, or control was mixed with the cationic lipid DOTAP as the manufacturer indicated (Roche Life Science, catalog 11020375001). After mixing for 5 seconds and incubation at $37^{\circ} \mathrm{C}$ for 15 minutes, the mixture $(0.5 \mathrm{nmol}$ in $5 \mu \mathrm{l})$ was injected into lateral ventricles. All agomirs and antagomirs were injected 3 times over a 7-day period before tests. Chemical drugs were injected s.c. once daily in this 7-day period for subsequent behavioral tests. For combining with AAV, 
mice were injected with AAVs as mentioned above. Three weeks later, miRNA agomirs and antagomirs were injected.

Quantitative real-time PCR. Mice were deeply anesthetized with pentobarbital and decapitated. The $\mathrm{MPFC}$ was removed immediately. Total RNA was isolated using the mirVana RNA Isolation Kit (Life Technologies). The iScript kit (Bio-Rad) was used to synthesize cDNA from mRNA, and the miRNA First-Strand cDNA Synthesis Kit (Agilent) was used to synthesize cDNA from miRNA. The experiments were performed using SYBR Green (Life Technologies).

The following forward primers were used for miRNA analysis: stem-loop reverse transcriptase primers: miR-221-5p, 5'-CTCAACTGGTGTCGTGGAGTCGGCAATTCAGTTGAGACAGAAAT-3'; miR541-3p， 5'-CTCAACTGGTGTCGTGGAGTCGGCAATTCAGTTGAGAGTATGGA-3'; quantitative real-time PCR primers: miR-221-5p, 5'-ACACTCCAGCTGGGACCTGGCATACAATGTAGAT-3'; miR-5413p, 5'-ACACTCCAGCTGGGTGGCGAACACAGAATC-3'; unified reverse primer: 5'-TGGTGTCGTGGAGTCG-3'; U6 forward, 5'CTCGCTTCGGCAGCACA-3'; U6 reverse, 5'-AACGCTTCACGAATTTGCGT-3'.

The following primers were used for mRNA analysis: Cnr1 forward, 5'-GAACCTGCTGGTGCTATGTGT-3'; Cnr1 reverse, 5'GGACTATCTTTGCGGTGGAAC-3'; Prl forward, 5'-CCAGAAAGCAGGGACACTCCTC-3'; Prl reverse, 5'-CCACACGGTCAAACAGCTCTC-3'; Gapdh forward, 5'-GGTTGTCTCCTGCGACTTCA-3'; Gapdh reverse, 5'-TGGTCCAGGGTTTCTTACTCC-3'.

The relative ratio of miRNA and mRNA for each sample was calculated from the threshold cycles using a software program (StepOne, $\mathrm{ABI}$ ) according to the manufacturer's instructions. U6 was used as a housekeeping gene to normalize miRNA expression. Endogenous Gapdh gene levels were used as a control.

Immunofluorescence staining. Brains were removed and postfixed in $4 \%$ paraformaldehyde overnight at $4^{\circ} \mathrm{C}$. Free-floating coronal sections $(20 \mu \mathrm{m})$ were obtained using a freezing microtome (CM1950, Leica). Prefrontal cortex sections containing the $\mathrm{MPFC}$ were washed in 0.1 $\mathrm{mM}$ PBS buffer and permeabilized with $0.3 \%$ Triton in $5 \%$ normal goat serum for 1 hour. Then sections were incubated in primary antibodies (Cell Signaling Technology, D5N5C; $1: 200$ ) overnight at $4^{\circ} \mathrm{C}$ in $10 \%$ normal goat serum. After washing, sections were incubated with secondary Cy3-conjugated anti-rabbit antibody (1:200; CWBIOTECH) for 2 hours at room temperature. Diluted DAPI in 0.1 mM PBS (1:1000) was applied to sections after washing for 5 minutes to stain nuclei. Sections were mounted onto slides using $50 \%$ glycerinum. Slides were observed using a confocal laser microscope (FV1000, Olympus), and images were captured by Fluoview 1000 (Olympus). Image analyses and processing were carried out using Image (NIH) by researchers blinded to the experiments.

Western blot analysis. Western blot analysis was performed as described previously (52). PVDF membranes (Millipore) were probed with polyclonal rabbit anti-mouse CB1 (Cell Signaling Technology, D5N5C; 1:1000), polyclonal rabbit anti-mouse CB2 (Abcam, ab3561; 1:1000), anti-PSD95 (Abcam, ab18258; 1:1000), polyclonal rabbit antimouse synaptophysin (Abcam, ab32127; 1:1000), polyclonal rabbit antimouse Neurl1a (Proteintech Group, 18898-1-AP; 1:500), polyclonal rabbit anti-mouse Neurl1b (Abcam, ab156988; 1:1000), monoclonal rabbit anti-mouse ubiquitin (Abcam, ab134953; 1:1000), monoclonal rabbit anti-mouse p-GluA1 $1_{\text {Ser845 }}$ (Abcam, ab76321; 1:1000), monoclonal rabbit anti-mouse $\mathrm{p}-\mathrm{GluA1} 1_{\text {Ser831 }}$ (Abcam, ab109464; 1:1000), monoclonal rab- bit anti-mouse ER $\alpha$ (Cell Signaling Technology, D8H8; 1:1000), monoclonal rabbit anti-mouse ER $\beta$ (Abcam, ab32063; 1:1000), polyclonal rabbit anti-mouse HSD17B1 (Abcam, ab217851; 1:1000), polyclonal rabbit anti-mouse HSD17B2 (Abcam, ab196784; 1:1000), monoclonal rabbit anti-mouse GluN2A (Abcam, ab124913; 1:1000), monoclonal rabbit anti-mouse GluN2B (Abcam, ab65783; 1:1000), monoclonal rabbit antimouse GluN1 (Cell Signaling Technology, D65B7; 1:1000), monoclonal rabbit anti-mouse GluA1 (Abcam, ab109450;1:1000), polyclonal rabbit anti-mouse GABA $\alpha 2$ (Abcam, ab72445; 1:1000), polyclonal rabbit antimouse GABAß2 (Abcam, ab156000; 1:1000), monoclonal rabbit antimouse FAAH (Abcam, ab128917; 1:1000), polyclonal rabbit anti-mouse MGLL (Abcam, ab24701; 1:1000), monoclonal rabbit anti-mouse DAGL $\alpha$ (Cell Signaling Technology, D3G8H; 1:1000), monoclonal rabbit anti-mouse DAGL $\beta$ (Cell Signaling Technology, D4P7C; 1:1000), and anti- $\beta$-actin (Sigma-Aldrich, A2228; 1:10,000). For data quantification, band intensity was expressed relative to the loading control ( $\beta$-actin). Membranes were incubated with horseradish peroxidase-conjugated secondary antibodies (anti-rabbit/anti-mouse IgG for the primary antibodies), and bands were visualized using an ECL system (Absin Bioscience Inc.). Band density was measured with a Tanon5200 imager.

Postsynaptic density fraction preparation. Postsynaptic density fraction of mouse cortex was prepared according to reported protocol (63).

Virus-mediated gene expression and stereotactic injection. For CB1 overexpression, AAVs were generated from the AAV-CMV-mCherryP2A-Cnr1-3Flag (AAV-Cnr1-mCherry) plasmid and produced commercially (OBiO; serotype 2/8; $4 \times 10^{12}$ infectious units per ml). For AAVmediated knockdown of endogenous Neurl1a and Neurl1b expression, engineered AAV coexpressing green fluorescent protein (GFP) under the CMV promoter and shRNA against mouse Neurl1 $a$ and Neurl1b or control shRNA under the U6 promoter were also produced by OBiO. The following shRNA sequences were used: Neurl1 $a, 5^{\prime}$-GGUGCUCAUCUACGAGCAATT-3'; Neurl1b, 5'-GCGAUUCAGACAUGACCUUTT-3'; scrambled, 5'-TTCTCCGAACGTGTCACGT-3'.

For stereotactic injection, mice were anesthetized with gaseous isoflurane. AAVs (more than $4 \times 10^{9}$ infectious viral particles per 1 $\mu \mathrm{l})$ were stereotaxically microinjected into the bilateral $\mathrm{mPFC}$ area (anteroposterior, $+2.43 \mathrm{~mm}$; mediolateral, $\pm 0.28 \mathrm{~mm}$; dorsoventral, $-1.81 \mathrm{~mm}$ ) at a rate of $0.1 \mu \mathrm{l} / \mathrm{min}$ for 10 minutes per side. The hole was sealed with bone wax. Mice were used for further experiments 3 weeks after injection. Viral manipulations of CB1, Neurl1a, and Neurl1b were confirmed by Western blot.

Luciferase assays. Wild-type and mutant 3'-UTRs of Neurl1a and Neurl1 $b$ containing miRNA-binding sites were synthesized by Sangon Biotech. HEK293T cells were plated at approximately 50\%-60\% confluence and transfected with miR-221-5p mimics as well as the $3^{\prime}$-UTR luciferase vector using Lipofectamine 3000 transfection reagent according to the manufacturer's instructions. Forty-eight hours after transfection, luciferase assay was performed using a dual luciferase reporter assay kit (Promega, catalog PR-E1910) in compliance with the manufacturer's instructions.

Coimmunoprecipitation. $\mathrm{MPFC}$ was rapidly removed, washed in ice-cold PBS, and lysed in buffer containing $50 \mathrm{mM}$ Tris- $\mathrm{HCl}$ ( $\mathrm{pH} 7.4$ ), $1 \%$ NP-40, 0.25\% sodium deoxycholate, $150 \mathrm{mM} \mathrm{NaCl}, 1 \mathrm{mM}$ EDTA, and protease inhibitors. Equal amounts of protein were incubated with antibodies overnight at $4^{\circ} \mathrm{C}$. Then, resuspended protein $\mathrm{A} / \mathrm{G}$ magnetic agarose beads (Thermo Fisher Scientific, catalog 78610) were added to each sample and incubated 2 hours at $4^{\circ} \mathrm{C}$. Beads were washed 3 
times with lysis buffer and resuspended in SDS sample buffer. Samples were separated on $8 \%-12 \%$ SDS-PAGE gels, and specific proteins were measured by immunoblotting.

Women's hsa-miRNAs and quantification. Women's blood samples were obtained from volunteers who intended to take a health examination in Healthcare Management Center, Xijing Hospital, Fourth Military Medical University. Women, aged 25-65 years, claimed no immunologic or neuropsychiatric disorders. Exclusion criteria included history of acute or chronic mental illness, history of gynecologic or breast cancer, use of psychotropic medications, contraindications to hormone therapy, and use of hormone therapy during the 3 months before their recruitment. Ages, time since the last menstrual period, and perimenopausal syndrome were recorded. Venous blood samples were taken at 8-9 am from forearm chelidon. The participants were asked not to take in any food for at least 12 hours before harvesting of blood samples. Blood sample $(10 \mathrm{ml})$ was collected in a $\mathrm{K}_{2}$ EDTA-containing tube and immediately centrifuged at $1000 \mathrm{~g}$ for 10 minutes at room temperature. Plasma was immediately frozen at $-80^{\circ} \mathrm{C}$ for approximately 1 week before extraction. miRNA was extracted from $1 \mathrm{ml}$ of plasma using the SanPrep Column microRNA Extraction Kit (Sangon Biotech, order B518811) according to the manufacturer's protocol except for noted modifications. The following forward primers were used for hsa-miRNA analysis: stem-loop reverse transcriptase primers: hsa-miR-221-5p, 5'-CTCAACTGGTGTCGTGGAGTCGGCAATTCAGTTGAGAAATCTAC-3'; hsa-miR541-3p, 5'-CTCAACTGGTGTCGTGGAGTCGGCAATTCAGTTGAGAGTCCAGA-3'; quantitative real-time PCR primers: hsa-miR-221-5p, forward, 5'-ACACTCCAGCTGGGACCTGGCATACAAT-3'; hsa-miR541-3p, forward, 5'-ACACTCCAGCTGGGTGGTGGGCACAGAA-3'; unified reverse primer, 5'-TGGTGTCGTGGAGTCG-3'; hsa-U6 forward, 5'-CTCGCTTCGGCAGCACA-3'; hsa-U6 reverse, 5'-AACGCTTCACGAATTTGCGT-3'.

p-cFos immunofluorescence staining. Ninety minutes after fear extinction training, mice were anesthetized with an overdose of sodium pentobarbital. mPFC slice preparation and staining were carried out as reported previously (58). p-cFos-positive cells were counted using ImageJ software (NIH; RRID: SCR_003070) by a rater blinded to the experimental condition.

ELISA. In plasma, estradiol is largely bound to sex hormone-binding globulin and albumin. Only a $2.21 \% \pm 0.04 \%$ fraction is free (64). ELISA was used to quantify levels of estrone (E1), $\beta$-estradiol (E2), estriol (E3), and 2-AG. Total levels of E1, E2, and E3 were detected by kits (catalog KA1908, Abnova; catalog ADI-900-008, Enzo Biochem; catalog ADI900-100, Enzo Biochem, respectively) according to the manufacturers' instructions. Levels of non-protein-bound free estradiol in plasma were detected by kit (R\&D Systems Co., catalog KGE014). 2-AG in plasma was detected by kit (catalog 365639, USBiological).

Quantitative LC-MS/MS analysis of endocannabinoids. Levels of 2-AG and AEA in MPFC were detected by liquid chromatographytandem mass spectrometry (LC-MS/MS). Tissues of mPFC were freshly dissected 1 week after treatments and stored at $-80^{\circ} \mathrm{C}$ for analysis. Tissues were homogenized on ice in $1 \mathrm{ml}$ RIPA lysate with internal standards (2 ng AEA-d5 and 20 ng 2-AG-d5). The homogenization process was less than 5 minutes, and homogenates were kept on ice until organic extraction to minimize the ex vivo generation of endocannabinoids. Homogenates were centrifuged at 10,000 $g$ for $10 \mathrm{~min}$ utes. Supernatants were transferred to $12-\mathrm{ml}$ glass tubes, extracted after 20 minutes with TBME $(6 \mathrm{ml})$, and centrifuged $(2800 \mathrm{~g}$,
5 minutes, at room temperature). Organic phase was transferred to clean tubes and evaporated $\left(40^{\circ} \mathrm{C}, 20\right.$ minutes) under a stream of nitrogen, and extracts were reconstituted in $1 \mathrm{ml}$ of a mixture (water/ methanol, 3:7 in vol/vol) and transferred to HPLC vials. Sample (20 $\mu \mathrm{l})$ was injected into an LC-MS/MS system. The liquid chromatography system used for analysis was Agilent 1200 HPLC (Agilent Technologies). Autosampler was kept at $12^{\circ} \mathrm{C}$. Liquid chromatography was performed on a Zorbax SB-CN $2.1 \times 100 \mathrm{~mm}, 3.5-\mu \mathrm{m}$ column (Agilent Technologies). Mobile phase A consisted of $10 \mathrm{mM}$ ammonium acetate. Mobile phase B consisted of $100 \%$ methanol. Gradient elution was performed at a flow rate of $300 \mu \mathrm{l} / \mathrm{min}$. Initial composition of the gradient was $45 \%$ $\mathrm{B}$ and was held isocratically for the first half-minute. Mobile phase B increased to $70 \% \mathrm{~B}$ within 0.5 minutes. From 1 to 10 minutes, the gradient ramped linearly from $70 \% \mathrm{~B}$ to $99 \% \mathrm{~B}$, where it was held for 4 minutes. At 14.5 minutes, the gradient returned to initial conditions of $45 \% \mathrm{~B}$ to re-equilibrate the column. Total run time was 21 minutes. The HPLC system was connected to an AB SCIEX QTRAP 4500 triple-quadrupole mass spectrometer. Electrospray mass spectrometry in positive mode conditions with source parameter optimization including cone voltage and positioning was optimized for quantification of analytes. Nebulizer and sheath gas flow rates of 10 and $81 / \mathrm{min}$ were used with source and sheath gas temperatures of $325^{\circ} \mathrm{C}$ and $250^{\circ} \mathrm{C}$, respectively. Peaks of analytes were integrated and quantified using MassHunter Workstation Software Quantitative Analysis (Agilent Technologies).

SUnSET for measurement of CB1 protein synthesis. SUnSET was performed as described previously (28). Transverse slices $(400 \mu \mathrm{m})$ containing $\mathrm{mPFC}$ were cut from sham, $\mathrm{OVX}_{\mathrm{ST}}$, and $\mathrm{OVX}_{\mathrm{LT}}$ mice as described previously (58). Slices were allowed to recover in artificial cerebrospinal fluid for 30 minutes at room temperature and then at $32^{\circ} \mathrm{C}$ for 2 hours. Puromycin $(5 \mu \mathrm{g} / \mathrm{ml}$; Selleck, catalog S7417) and/ or E2 (1 nM; Sigma-Aldrich, catalog E8875) was applied to slices for 1 hour at $32^{\circ} \mathrm{C}$ to label newly synthesized proteins. Slices were snapfrozen on dry ice, lysed, and homogenized. Protein lysate $(1 \mathrm{mg} / \mathrm{ml})$ was incubated with primary antibody (anti-puromycin, Merck Millipore, MABE343, 1:5000; anti-CB1, Cell Signaling Technology, D5N5C, 1:50) and protein A/G magnetic agarose beads (Thermo Fisher Scientific, catalog 78610) for immunoprecipitation. Amount of newly synthesized protein was detected by band intensity.

Statistics. Data were expressed as mean \pm SEM. Statistical comparisons were performed by either unpaired Student's $t$ test or 1-way or 2-way 2-sided ANOVA. If ANOVA was significant, post hoc comparisons were followed by Bonferroni's test. Repeated-measurement data were analyzed by univariate ANOVA after lower-bound correction. A $P$ value less than 0.05 was considered statistically significant.

Study approval. The animal experimental procedures were approved by the Institutional Animal Care and Use Committee of the Fourth Military Medical University. Women's blood samples were obtained from Xijing Hospital. The study was approved by the Xijing Hospital ethics committee, and written informed consent was obtained from all subjects.

\section{Author contributions}

$\mathrm{KZ}$ performed experiments, analyzed data, and wrote the paper. QY, LY, Yan-JL, XSW, Yu-JL, RLD, SYG, YYG, TS, YMW, AL, and YZ performed experiments and analyzed data. SBL and MGZ designed the experiments and wrote the paper. All authors approved the manuscript. 


\section{Acknowledgments}

This research was supported by the National Natural Science Foundation of China (31771119 to MGZ and 81771420 to SBL). The authors thank Min Zhuo (University of Toronto) for assistance with MED64 recording analysis.
Address correspondence to: Ming-gao Zhao or Shui-bing Liu, Department of Pharmacy, Precision Pharmacy and Drug Development Center, Tangdu Hospital, Fourth Military Medical University, Xinsi Road 1, Xi'an, Shaanxi, 710038, China. Phone: 86.29.84774552; Email:minggao@fmmu.edu.cn (MZ); liushb@fmmu.edu.cn (SL).
1. Greendale GA, Lee NP, Arriola ER. The menopause. Lancet. 1999;353(9152):571-580.

2. Luine VN. Estradiol and cognitive function: past, present and future. Horm Behav. 2014;66(4):602-618.

3. Rapp SR, et al. Effect of estrogen plus progestin on global cognitive function in postmenopausal women. The Women's Health Initiative Memory Study: a randomized controlled trial. JAMA. 2003;289(20):2663-2672.

4. Sherwin BB. Estrogen and memory in women: how can we reconcile the findings? Horm Behav. 2005;47(3):371-375.

5. Smith CC, Vedder LC, Nelson AR, Bredemann TM, McMahon LL. Duration of estrogen deprivation, not chronological age, prevents estrogen's ability to enhance hippocampal synaptic physiology. Proc Natl Acad Sci U S A. 2010;107(45):19543-19548.

6. Bouton ME. Context and behavioral processes in extinction. Learn Mem. 2004;11(5):485-494.

7. Bouton ME. Context, time, and memory retrieval in the interference paradigms of Pavlovian learning. Psychol Bull.1993;114(1):80-99.

8. Myers KM, Davis M. Mechanisms of fear extinction. Mol Psychiatry. 2007;12(2):120-150.

9. Gale GD, et al. Role of the basolateral amygdala in the storage of fear memories across the adult lifetime of rats. J Neurosci. 2004;24(15):3810-3815.

10. Maren S, Holmes A. Stress and fear extinction. Neuropsychopharmacology. 2016;41(1):58-79.

11. Davis M, Myers KM. The role of glutamate and gamma-aminobutyric acid in fear extinction: clinical implications for exposure therapy. Biol Psychiatry. 2002;52(10):998-1007.

12. Marsicano G, et al. The endogenous cannabinoid system controls extinction of aversive memories. Nature. 2002;418(6897):530-534.

13. Bartel DP. MicroRNAs: genomics, biogenesis, mechanism, and function. Cell.2004;116(2):281-297.

14. McNeill E, Van Vactor D. MicroRNAs shape the neuronal landscape. Neuron. 2012;75(3):363-379.

15. Hernandez-Rapp J, Rainone S, Hébert SS. MicroRNAs underlying memory deficits in neurodegenerative disorders. Prog Neuropsychopharmacol Biol Psychiatry. 2017;73:79-86.

16. Follert P, Cremer H, Béclin C. MicroRNAs in brain development and function: a matter of flexibility and stability. Front Mol Neurosci. 2014;7:5.

17. Rao YS, Mott NN, Wang Y, Chung WC, Pak TR. MicroRNAs in the aging female brain: a putative mechanism for age-specific estrogen effects. Endocrinology. 2013;154(8):2795-2806.

18. Klinge CM. miRNAs regulated by estrogens, tamoxifen, and endocrine disruptors and their downstream gene targets. Mol Cell Endocrinol. 2015;418(pt 3):273-297.

19. Collingridge GL, Peineau S, Howland JG, Wang YT. Long-term depression in the CNS. Nat Rev Neurosci. 2010;11(7):459-473.

20. Gilmartin MR, Helmstetter FJ. Trace and contextual fear conditioning require neural activity and NMDA receptor-dependent transmission in the medial prefrontal cortex. Learn Mem. 2010;17(6):289-296.

21. Cho JH, Deisseroth K, Bolshakov VY. Synaptic encoding of fear extinction in MPFC-amygdala circuits. Neuron. 2013;80(6):1491-1507.

22. Harris KM, Jensen FE, Tsao B. Three-dimensional structure of dendritic spines and synapses in rat hippocampus (CA1) at postnatal day 15 and adult ages: implications for the maturation of synaptic physiology and long-term potentiation. J Neurosci. 1992;12(7):2685-2705.

23. Hotulainen P, Hoogenraad CC. Actin in dendritic spines: connecting dynamics to function. JCell Biol. 2010;189(4):619-629.

24. Connor SA, Wang YT. A place at the table: LTD as a mediator of memory genesis. Neuroscientist. 2016;22(4):359-371.

25. Castillo PE, Younts TJ, Chávez AE, Hashimotodani Y. Endocannabinoid signaling and synaptic function. Neuron. 2012;76(1):70-81.

26. Koch M, et al. Hypothalamic POMC neurons promote cannabinoid-induced feeding. Nature. 2015;519(7541):45-50.

27. Berry KP, Nedivi E. Spine dynamics: are they all the same? Neuron. 2017;96(1):43-55.

28. Schmidt EK, Clavarino G, Ceppi M, Pierre P. SUnSET, a nonradioactive method to monitor protein synthesis. Nat Methods. 2009;6(4):275-277.

29. Koutelou E, et al. Neuralized-like 1 (Neurl1) targeted to the plasma membrane by N-myristoylation regulates the Notch ligand Jagged1. J Biol Chem. 2008;283(7):3846-3853.

30. Wu G, Liu C, He X. Ozz; a new name on the long list of beta-catenin's nemeses. Mol Cell. 2004;13(4):451-453.

31. Rullinkov G, et al. Neuralized-2: expression in human and rodents and interaction with Delta-like ligands. Biochem Biophys Res Commun. 2009;389(3):420-425.

32. Alvarado-García A, et al. [Clinical practice guideline. Diagnosis and treatment of postmenopausal and perinemopausia]. Rev Med Inst Mex Seguro Soc. 2015;53(2):214-225.

33. Sherwin BB. Estrogen and cognitive aging in women. Neuroscience. 2006;138(3):1021-1026.

34. Maki PM, Zonderman AB, Resnick SM. Enhanced verbal memory in nondemented elderly women receiving hormone-replacement therapy. Am J Psychiatry. 2001;158(2):227-233.

35. Resnick SM, Metter EJ, Zonderman AB. Estrogen replacement therapy and longitudinal decline in visual memory. A possible protective effect? Neurology. 1997;49(6):1491-1497.

36. Shumaker SA, et al. Conjugated equine estrogens and incidence of probable dementia and mild cognitive impairment in postmenopausal women: Women's Health Initiative Memory Study. JAMA. 2004;291(24):2947-2958.
37. Resnick SM, Coker LH, Maki PM, Rapp SR, Espeland MA, Shumaker SA. The Women's Health Initiative Study of Cognitive Aging (WHISCA): a randomized clinical trial of the effects of hormone therapy on age-associated cognitive decline. Clin Trials. 2004;1(5):440-450.

38. Gleason CE, et al. Effects of hormone therapy on cognition and mood in recently postmenopausal women: findings from the randomized, controlled KEEPS-Cognitive and Affective Study. PLoS Med. 2015;12(6):e1001833 .

39. Espeland MA, et al. Long-term effects on cognitive function of postmenopausal hormone therapy prescribed to women aged 50 to 55 years. JAMA Intern Med. 2013;173(15):1429-1436.

40. Henderson VW, et al. Cognitive effects of estradiol after menopause: a randomized trial of the timing hypothesis. Neurology. 2016;87(7):699-708.

41. Sherwin BB. Estrogen and/or androgen replacement therapy and cognitive functioning in surgically menopausal women. Psychoneuroendocrinology. 1988;13(4):345-357.

42. Kindt M, Soeter M. Reconsolidation in a human fear conditioning study: a test of extinction as updating mechanism. Biol Psychol. 2013;92(1):43-50.

43. Neves G, Cooke SF, Bliss TV. Synaptic plasticity, memory and the hippocampus: a neural network approach to causality. Nat Rev Neurosci. 2008;9(1):65-75.

44. Dalton GL, Wang YT, Floresco SB, Phillips AG. Disruption of AMPA receptor endocytosis impairs the extinction, but not acquisition of learned fear. Neuropsychopharmacology. 2008;33(10):2416-2426.

45. Bhardwaj SK, Tse YC, Ryan R, Wong TP, Srivastava LK. Impaired adrenergic-mediated plasticity of prefrontal cortical glutamate synapses in rats with developmental disruption of the ventral hippocampus. Neuropsychopharmacology. 2014;39(13):2963-2973.

46. Gerdeman GL, Ronesi J, Lovinger DM. Postsynaptic endocannabinoid release is critical to longterm depression in the striatum. Nat Neurosci. 2002;5(5):446-451.

47. Min R, Nevian T. Astrocyte signaling controls spike timing-dependent depression at neocortical synapses. Nat Neurosci. 2012;15(5):746-753.

48. Qiu DL, Knöpfel T. Presynaptically expressed long-term depression at cerebellar parallel fiber synapses. Pflugers Arch. 2009;457(4):865-875.

49. Castelli MP, et al. Male and female rats differ in brain cannabinoid $\mathrm{CB} 1$ receptor density and function and in behavioural traits predisposing to drug addiction: effect of ovarian hormones. Curr Pharm Des. 2014;20(13):2100-2113.

50. Guan JS, Xie H, Ding X. The role of epigenetic regulation in learning and memory. Exp Neurol. 2015;268:30-36.

51. Michalon A, et al. Chronic pharmacological mGlu5 inhibition corrects fragile $\mathrm{X}$ in adult mice. Neuron. 2012;74(1):49-56. 
52. Yang Q, et al. Increased coupling of caveolin-1 and estrogen receptor $\alpha$ contributes to the fragile X syndrome. Ann Neurol. 2015;77(4):618-636.

53. Zhang $\mathrm{K}$, et al. Imbalance between $\mathrm{TNF} \alpha$ and progranulin contributes to memory impairment and anxiety in sleep-deprived mice. Sci Rep. 2017;7:43594.

54. Tian Z, et al. Estrogen receptor GPR30 exerts anxiolytic effects by maintaining the balance between GABAergic and glutamatergic transmission in the basolateral amygdala of ovariectomized mice after stress. Psychoneuroendocrinology. 2013;38(10):2218-2233.

55. Feng B, et al. Anxiolytic actions of motilin in the basolateral amygdala. Mol Neurobiol. 2013;47(3):892-902.
56. Lucas G, et al. Serotonin(4) (5-HT(4)) receptor agonists are putative antidepressants with a rapid onset of action. Neuron. 2007;55(5):712-725.

57. Liu MG, et al. Long-term potentiation of synaptic transmission in the adult mouse insular cortex: multielectrode array recordings. J Neurophysiol. 2013;110(2):505-521.

58. Zhang K, et al. Elevated progranulin contributes to synaptic and learning deficit due to loss of fragile X mental retardation protein. Brain. 2017;140(12):3215-3232.

59. Trapnell C, Pachter L, Salzberg SL. TopHat: discovering splice junctions with RNA-Seq. Bioinformatics. 2009;25(9):1105-1111.

60. Trapnell C, et al. Differential gene and transcript expression analysis of RNA-seq experi- ments with TopHat and Cufflinks. Nat Protoc. 2012;7(3):562-578.

61. Lee ST, et al. miR-206 regulates brain-derived neurotrophic factor in Alzheimer disease model. Ann Neurol. 2012;72(2):269-277.

62. Gehrke S, Imai Y, Sokol N, Lu B. Pathogenic LRRK2 negatively regulates microRNA-mediated translational repression. Nature. 2010;466(7306):637-641.

63. Tao Y, et al. Erbin interacts with TARP $\gamma$-2 for surface expression of AMPA receptors in cortical interneurons. Nat Neurosci. 2013;16(3):290-299.

64. Wu CH, Motohashi T, Abdel-Rahman HA, Flickinger GL, Mikhail G. Free and protein-bound plasma estradiol-17 $\beta$ during the menstrual cycle. J Clin Endocrinol Metab. 1976;43(2):436-445. 